\title{
MQSMER: A Mixed Quadratic Shape Model with Optimal Fuzzy Membership Functions for Emotion Recognition
}

\author{
R. Vishnu Priya ${ }^{\mathrm{a}}$, V. Vijayakumar ${ }^{\mathrm{a}}$, João Manuel R. S. Tavares ${ }^{\mathrm{b}, *}$ \\ a School of Computing Science and Engineering, VIT University, Chennai Campus, Chennai, \\ Tamilnadu, India

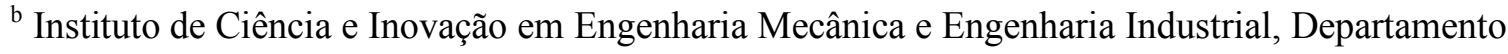 \\ de Engenharia Mecânica, Faculdade de Engenharia, Universidade do Porto, Porto, Portugal
}

\begin{abstract}
The traditional geometrical based approaches used in facial emotion recognition fail to capture the uncertainty present in the quadrilateral shape of emotions under analysis, which reduces the recognition accuracy rate. Furthermore, these approaches require extensive computational time to extract the facial features and to train the models. This article proposes a novel geometrical fuzzy based approach to accurately recognize facial emotions in images in less time. The four corner vertices of the mouth are the most important features to recognize facial emotions and can be extracted without the need of a reference face. These extracted features can then be used to define the quadrilateral shape, and the associated degree of impreciseness in the shape can be accessed using the proposed geometric fuzzy membership functions. Hence, four fuzzy features are derived from the membership functions and given to classifiers for emotion evaluations. In our tests, the fuzzy features achieved an accuracy rate of $96.17 \%$ in the Japanese Female Facial Expression database, and 98.32\% in the Cohn-Kanade Facial Expression database, which are higher than the ones achieved by other common up-to-date methods. In terms of computational time, the proposed method required an average of 0.375 seconds to build the used model in a common PC.
\end{abstract}

Keywords: Emotion Recognition; Fuzzy-Shape; Geometric Features; Facial Expression.

\footnotetext{
* Corresponding author. Tel.: +351 220413472; Fax: +351 225081445 (João Manuel R. S. Tavares).

Email addresses: vishnupriya.r@vit.ac.in (R. Vishnu Priya), vijayakumar.v@vit.ac.in (V. Vijayakumar), tavares@fe.up.pt (João Manuel R. S. Tavares).
} 


\section{Introduction}

Emotions play an important role in our daily lives. A study on communications through emotions conducted by a psychologist (Mehrabian 1968) found that $55 \%$ of our usual messages are transmitted through facial expressions or emotions, vocal cues convey $38 \%$ and the remainder $7 \%$ is expressed using verbal cues. This suggests that facial expressions play a major role in human social interactions. Typically, facial expressions are created through shrinking of one or more facial muscles, which temporarily deform facial components. Ekman and Friesen (1978) developed a well-accepted study on facial expressions and suggested that expressions are universal across human ethnicities and cultures. Their research also stated that there are six basic emotions: anger, disgust, fear, happiness, sadness and surprise, which can be evaluated based on facial muscle movements generated by 44 anatomical Action Units (AUs) defined in the Facial Action Coding System (FACS). In recent years, several authors managed to recognize facial emotions using AUs (Zhang L et al. 2015; Jain s et al. 2011; Wu T et al. 2010; Shan C et. 2009). However, it is a very laborious task to determine emotions using the FACS; consequently, attention has been given to automatic recognition of emotions. The recent progress in automation has seen a fast growth in facial expression analysis with applications in computer vision, pattern recognition and Human-Computer Interaction. Several other applications, such as Emo chat (Anderson \& McOwan 2004), intelligent tutoring system (Whitehill et al. 2008), facial animation, and virtual reality of facial emotions have also been developed for the recognition of emotions. Systems for automatic detection of facial expressions can extract relevant facial features from either static images or image sequences that are input to computational classifiers to recognize the respective emotions. Usually, there are two ways to recognize facial expressions, namely, geometric based and appearance based approaches. The geometric based approach uses the shape and position of the face under analysis, while the appearance based features approach uses wrinkles, bulges, furrows, and other facial peculiarities, and obtain essential information about facial expressions through micro-patterns. Several appearance based algorithms have been proposed (Happy S L et al. 2015; Poursaberi. A et 
al. 2012; Zhong et al. 2012; Zhang L et al. 2011; Mingli song M et al. 2010; Uddin M Z et al. 2009). However, the major challenge of appearance based features is its inability to generalize appearance based features across different human races. Although geometric features also have their drawbacks, for example, they are very difficult to track and can easily be affected by noise, they can generate all the necessary information to recognize facial expressions (Valstar et al. 2005). In fact, humans have an extraordinary ability to recognize expressions, and, for example, even when a cartoon image has only facial contours, they can easily recognize the associated expression ( $\mathrm{Gu}$ et al. 2010). Therefore, geometric based features seem to be the best option for the development of computational systems to recognize human expressions.

Most of the algorithms in the literature to detect facial expressions accurately can be classified as holistic or local. Eigenfaces and Fisherfaces (Turk and Pentland. 1991; Belhumeur et al. 1997) are holistic methods that extract facial features from the complete face under analysis On the other hand, local methods separate a face image into a few small blocks and apply certain feature extraction algorithms. The authors in (Heisele et al. 2007; Zou et al. 2007) reported that the performance of facial expression recognition is significantly increased when local features are used compared to the whole face. Those local descriptors are identified through deformations of eyebrows, eyes, nose, mouth and 42 muscles. Among the local regions, Li et al. (2013) stated that expression recognition based on the mouth is more rewarding than one based on the upper part of the face, that is the eyes. This statement can be justified since: First, the extraction of feature points from the mouth is easier than from the eye because the feature points in the mouth are much more clearly distinguished from each other. Majunder et al. (2014) reported that the feature detection in the eyes is a challenging task due to the presence of eyelashes, shadows between the eyes and eyebrows, and the very small gap between eyes and eyebrows. Moreover, the eye vertices are located in the skin region without a distinctive grey scale. Second, the main deformations in the face due to emotions are in the mouth region. Third, the main discerning features associated to facial expressions are distributed in the lower part of the face (Gu et al. 2012). Fourth, 
although it is well-known that the eye is highly sensitive to emotions, the stimulus response to the emotions is very small. Furthermore, each longitudinal section of the face seems to be a mirror of the other one, but the symmetrical view does not resemble the same, as can be observed in Figure 1. These findings clearly suggest that the full mouth region with its geometrical nature can generate promising results.

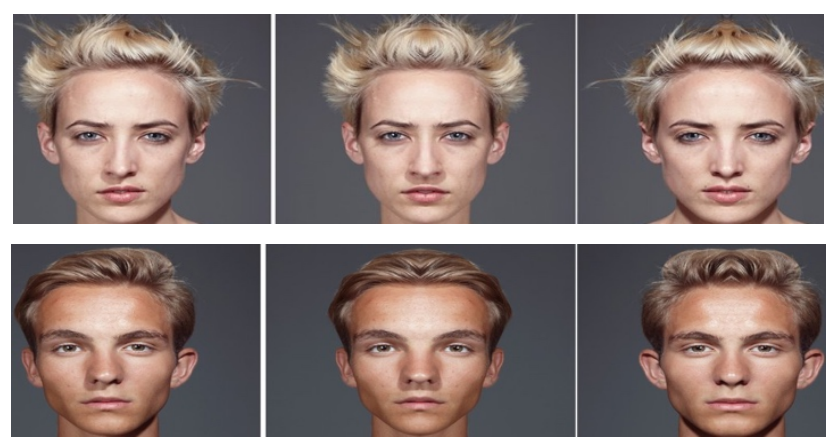

Figure 1 Two sample examples of the symmetrical view of the face: the face on the left is the original face and the face in the centre is the left symmetry and on the right is the right symmetry.

This work introduces a fully automatic method for facial expression recognition using geometric features. A set of four corner vertices is extracted from the mouth region of the static image under analysis. The extracted features are used to define the quadrilateral shape and are then processed by the proposed fuzzy membership functions. The fuzzy features are derived from the membership functions with the ability to deal with uncertainty and are processed by a classifier that recognizes the presence of any basic expression. The experimental results show that the proposed approach achieves high recognition rates. The flow chart of the proposed approach is shown in Figure 2. 


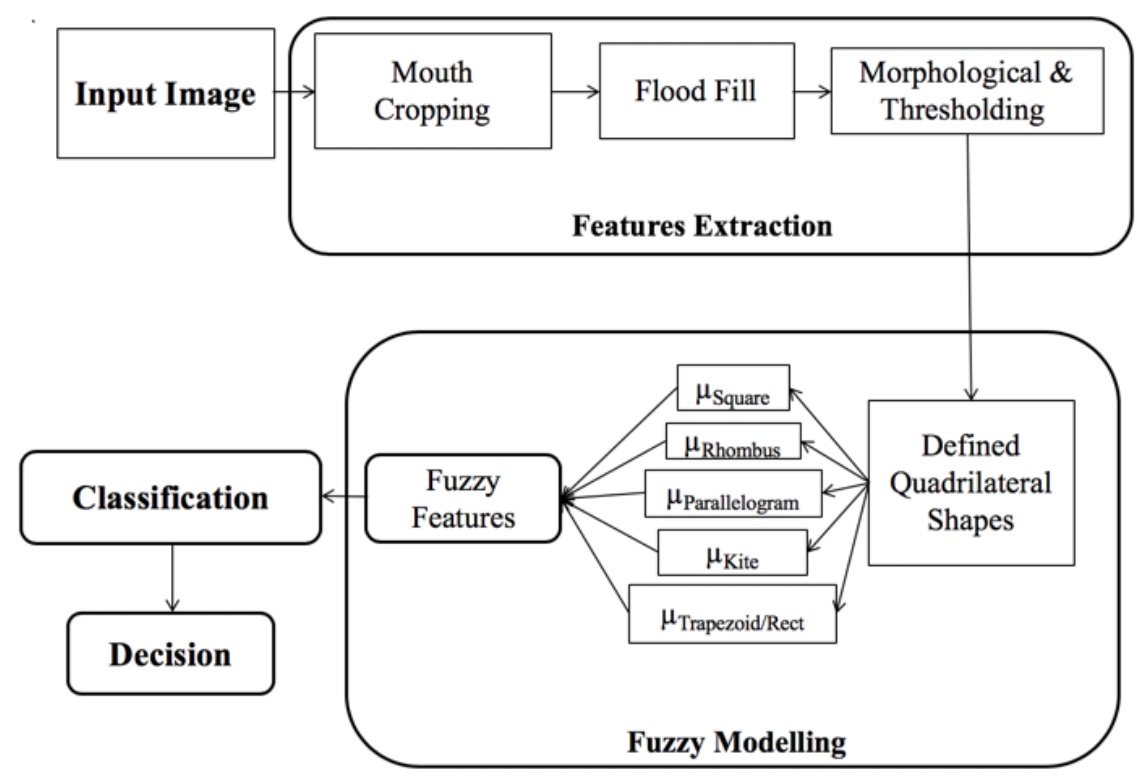

Figure 2 Flow chart of the proposed approach

This article is organized as follows: related works are reviewed in Section 2, the proposed approach is presented in Section 3; the results of the proposed approach are compared to the ones obtained by other up-to-date approaches in Section 4; and finally, Section 5 brings the conclusions and suggests future works.

\section{Related work}

Researchers have worked on human facial emotion recognition for several decades and various techniques and approaches to recognize emotions have been proposed. Some of these techniques and approaches are reviewed in the following subsections.

\subsection{Emotion recognition from whole faces}

To recognize emotions from whole faces, researchers have exploited pixel based information (Wang and Ruan, 2010; Rahulamathavan et al. 2013), Wavelet transform (Shih et al. 2008; Kazmi et al. 2012), Gabor filtering (Donato et al. 1999; Deng et al. 2005), edges and skin detection (Ilbeygi and Hosseini 2012), Discrete Cosine Transform (Kharat and Dudul 2009; Gupta et al. 2011), optical flow analysis (Anderson and McOwan 2006), thermal 
analysis (Sophie et al. 2011), local binary pattern (Feng et al. 2004; Liu et al. 2009; Moore and Bowden 2009; Shan et al. 2009; Zhao and Zhang 2011; Zhang X et al. 2012; Rizwan et al. 2013; Luo et al. 2013) and level set (Sohail et al. 2011) based methods. These methods extract features from whole faces of different persons, which increases the dimensionality of the recognition problem and the required computational time and complexity grows.

\subsection{Appearance-based approaches}

The major disadvantage of active based model methods, like the Active Appearance Model (AAM) (Xiaorong Pu et al. 2015; Luo et al. 2011) and the Active Shape Model (ASM) is the need for prior information concerning the expected shape features. During the training phase, the shape features of these models have to be identified, usually manually (Laniti et al. 1997), and the recognition rate also strongly depends on the sample set used for training. A recent study to recognize facial expressions addressed the problem through the selection of the region near salient facial components: the extraction and matching of salient patchbased Gabor features was suggested in (Zhang et al. 2011). However, the proposed appearance based method achieved low recognition rates due to the inefficiency in selecting suitable patches for matching. Gu et al. (2010) used a radial encoding strategy based on Gabor filters to recognize facial expressions. The self-organizing map was applied to check the homogeneity of the encoded contours. The experimental results obtained using faces without occlusion, i.e. whole faces, and with local occlusions, showed interesting results. Xie and ManLam (2009) introduced the shape and texture based method for facial expression recognition. Thiago et al. (2013) used a multi-objective genetic algorithm to select the best features from a pool built using Gabor filtering and local binary patterns. However, the selection of the more suitable features from the salient regions increased the required processing time.

\subsection{Geometric-based approaches}

In these approaches, the geometric features are extracted from areas of facial components, e.g. eyes, mouth and nose, and then the geometric relations among the extracted features are processed. Kobayashiand (1997) developed a local facial features model using geometric 
facial points. Zhang Z et al. (1998) suggested the used of the position of fiducial points of the face under analysis, the multi-scale and multi-orientation Gabor wavelet coefficients at the same points or their combination to address the problem of facial expression recognition. Several recent geometric based approaches are based on geometric feature tracking (Kotsia and Pitas 2007; Song et al. 2010; Valstar and Pantic 2012), Discriminant Non-negative Matrix Factorization (Kotsia et al. 2008), graph based feature point tracking (Zafeiriou and Pitas 2008) and facial contours (Gu, Venkatesh and Xiang, 2010). In a common approach, the deformation of facial components is assessed by tracking the variation of feature points from the expressive image under study to the related neutral image. Usually, humans have the ability to recognize facial expressions without any reference face. Hence, the development of solutions for facial expression recognition using reference faces reduces their success, as they are very different from the way humans perceive emotions, and also it increases the pre-processing time. Moreover, emotion analysis based on geometrical shapes always contains a certain level of ambiguity, which was not been taken into account in the previously mentioned approaches.

\subsection{Recognition Modules}

Various classifiers have been used to build recognition modules for facial expressions. The well-known recognition modules are based on support vector machines (SVMs), hidden Markov models (HMM), Random Forest, Boosting, Bagging, Gaussian mixture models (GMM), dynamic Bayesian networks (DBN), and MultiLayer Perceptron (MLP). For example, (Asthana et al., 2009, Ghimire D et al. 2013, Kotisa I et al, 2007, Moore S et al. 2011, Rudovic O et al. 2012, Saeed A et al. 2014, Zhang S et al., 2012, Bartlett 2005; Sarah Adel Bargal et al. 2016) used SVMs, HMM models were used in (Yeasin M et al., 2006, Uddin M et al. 2009), MLP based networks in (Zhang et al. 1999; Mayor Torres et al. 2017; Pawel Tarnowski et al. 2017), Deep Neural networks in (Wan Ding et al. 2016; Yuchi Huang et al. 2016; Pablo Barros et al. 2017), and Radial Basis Function Networks (RBFN) in (Rosenblum et al. 1996) to classify facial emotions directly, but always without taking into account the vagueness presented in the model, which can reduce the recognition rates. 
The above review shows that the recent approaches have failed to capture the ambiguity presented in the geometric shape under analysis. Also the deformations associated with the expression need to be found by relating them to a corresponding neutral facial image. This reduces the efficiency and increases the required computational time and complexity. In our approach, the reference image is not needed, and a reduced number of features are extracted from the mouth to be analysed. The extracted features are then used to define the quadrilateral shape for each emotion and the fuzzy membership functions are derived from the shape. The proposed fuzzy membership functions are a square, rhombus, kite and an isosceles trapezoid. These four fuzzy functions produce the fuzzy features to capture the impreciseness and vagueness, i.e. the uncertainty, present in the shape. Then, SVM and Random Forest based classifiers are used for recognition. The results show that the recognition rate of the proposed method is higher than the ones from other recent approaches found in the literature.

\section{Mixed Quadratic Shape Model}

Facial expression analysis is generally divided into three main phases: feature extraction, geometric transformation and expression classification. Here, the first phase concerns the detection and extraction of feature points. The challenging issue in this phase is to find the optimal number of feature points to be used. The maximum number of extracted feature points found in the literature was 185 (Zhang et al. 2011). However, the number of extracted features should be as low as possible in order to reduce computational times. In the other more common related works a facial reference image is needed, i.e. a face in a neutral state. Then reference features are extracted from the image for analysis. This causes an additional delay in the pre-processing stage and is also very different from the way humans perceive objects. Most of the recent works fail to discriminate emotions using traditional classification methods because impreciseness and vagueness present in the geometrical shapes are not captured. In this work, the aforesaid disadvantages are overcome by extracting a minimum number of feature points from the mouth and using the geometric fuzzy 
membership functions. The fuzzy features derived from fuzzy membership functions are used to classify the six basic emotions. The adopted fuzziness has the ability to deal with the uncertainty in shape that helps to effectively discriminate the emotions. The idea of the proposed mixed quadratic shape model (MQSM) developed to identify the emotions is described in the following subsections.

\subsection{Background}

A geometric-based approach can be used to describe the shape associated to a face. Some of the facial geometrical features commonly used in the literature are: point, line, triangle, circle, oval, ellipse and quadrilateral. However, to initialize and track facial shapes is challenging. Vadivel et al. (2015) tracked the oval shape of the mouth using 13 feature points, but tracking all the points along the border of a shape is a difficult and time consuming task. They also interconnected the centre point with the vertex points to measure the deformation involved, which requires extra computational time. Ghimire and Lee (2014) tracked 52 facial key features modelled on points and lines to recognize facial expressions. Saeed et al. (2014) used eight facial keypoints to model the geometric structure of the face. Recently, Deepak Ghimire et al. (2017) extracted 52 facial keypoints to develop their facial geometric model based on lines and triangles. They proved that the triangle based representation outperforms both line and point based representations. The triangle is half of a quadrilateral.

The proposed approach defines the quadrilateral shape from four vertices of the mouth. The defined shape failed to match the quadrilateral shapes in geometry due to the ambiguity involved in the defined shape; however, this is overcome by using the proposed fuzzy membership functions.

\subsection{Region of Interest}

As per discussion in the introduction, the mouth region has the highest deformation levels in faces due to emotions, therefore it is considered as the Region of Interest (ROI) in this work. Moreover, psychologically, the left half of the entire body is controlled by the right part of the brain and the right half is controlled by the left part of the brain. As per Nielsen $e t$ al. (2013) stated, the emotions are more expressive in the left half of the face of the people 
with right brain activity and vice-versa. Therefore, the emotions extracted from the full mouth region are more truthful. The poses of the mouth can be used to find the associated deformations as listed in Table 1 for different type of facial emotions (Barthomeuf et al. 2012). Based on Table 1, one can conclude that the left, upper, right and lower mouth vertices are the highlights for each emotion. Therefore, these four feature points of the mouth are employed in the current work. This low number of points reduces the required processing time, resource and storage space substantially, which facilitates, for example, the implementation in micro and nano electronic devices. The proposed approach is explained in the following subsections on a step-by-step basis.

Table 1. Emotions and respective mouth poses

\begin{tabular}{|c|c|}
\hline Emotion & Mouth Poses \\
\hline Fear & $\begin{array}{l}\text { Lip corners pulled sideways, tighten and elongating } \\
\text { the mouth }\end{array}$ \\
\hline Happy & Lips corners pulled up \\
\hline Anger & Lips tighten and pressed together \\
\hline Surprise & Mouth opened as jaw drops \\
\hline Disgust & $\begin{array}{l}\text { Mouth opened with upper lip raised, and tongue stuck } \\
\qquad \text { out }\end{array}$ \\
\hline Sadness & Lips corner pulled straight \\
\hline
\end{tabular}

\subsection{Feature Points Extraction}

The face to be analysed is localized in the input image and, to reduce the computational time, only three quarters of the lower part of the face is considered here as the ROI. Then, the mouth is cropped manually from the previous defined ROI; this results in the image $C(x, y)$ , Figure 3(a). Then the flood fill algorithm is applied to obtain the intensity values of dark regions that are enclosed by lighter regions to the same intensity level, and the enhanced image $C_{f f a l g o}(x, y)$ is obtained, Figure $3(\mathrm{~b})$. The latter image is further processed through thresholding and a morphological opening operation to obtain the contour boundaries: 
$T_{h}(x, y)=\sum_{i=0}^{x} \sum_{j=0}^{y} \begin{cases}1, & \text { if } C_{\text {ffalgo }}(x, y) \geq T, \\ 0, & \text { otherwise }\end{cases}$

$g(x, y)=\left(T_{h} \Theta S\right) \oplus S$

where $T$ is the global threshold and $S$ is the $3 \times 3$ structuring element. The contour boundary $g(x, y)$ is used to find the four vertices based on the $\min$ and $\max$ values of the ' $x$ ' and ' $y$ ' coordinates of its points, respectively. These four vertices are denoted as $A, B, C$ and $D$, which represent the left, right, top and bottom of the mouth, respectively:

$$
\begin{array}{ll}
A=g_{A}(x, y) ; & g_{A}(x)=\text { Min }, \forall y \text { and } \quad g_{A}(y)=\operatorname{Max}(\text { pixel value }) \\
B=g_{B}(x, y) ; & g_{B}(x)=\text { Max }, \forall y \text { and } g_{B}(y)=\operatorname{Max}(\text { pixel value }) \\
C=g_{c}(x, y) ; & g_{c}(x)=\operatorname{Max}(\text { pixel value }) \text { and } g_{c}(y)=\text { Max }, \forall x \\
D=g_{D}(x, y) ; & g_{D}(x)=\operatorname{Max}(\text { pixel value }) \text { and } g_{D}(y)=\text { Min }, \forall x
\end{array}
$$

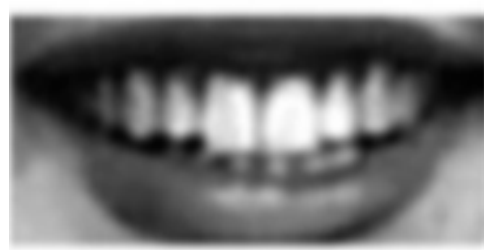

(a) $C(x, y)$

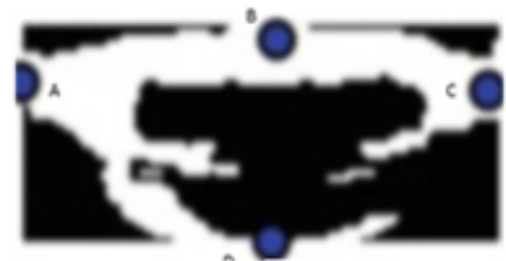

(b) $C_{\text {ffalgo }}(x, y) \& g(x, y)$

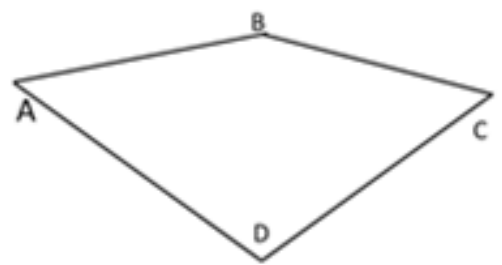

(c) Defined Quadrilateral Shape

Figure 3 Example of the low-level feature extraction from a mouth in an input image:

(a) Mouth segmented region; (b) Four mouth corner vertices; (c) Defined quadrilateral shape built for the mouth.

Using the four points $A, B, C$ and $D$, the quadratic shape is defined, as shown in Figure 2. Using this shape, different human emotions can be recognized.

\subsection{Quadrilateral Shape Definition}

Figure 4 shows the defined quadratic shapes of the mouth in the images indexed with 'KA.' from the Japanese Female Facial Expression (JAFFE) benchmark dataset, which 
contains 6 emotions: $E=\{$ angry, disgust, fear, happy, sad and surprise $\}$. The group of defined quadrilateral shapes for the $e^{\text {th }}$ emotion in Figure 4 is denoted as $G p D Q S^{L} e_{e}$, where $e \in\{1,2, \ldots E\}$ represents the emotion index and represents the group index, and a single quadrilateral in a group is denoted as $D Q S^{L}$.

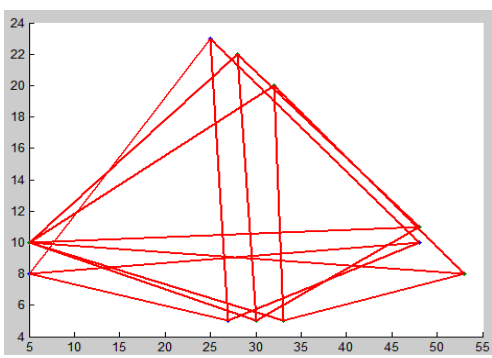

(a)

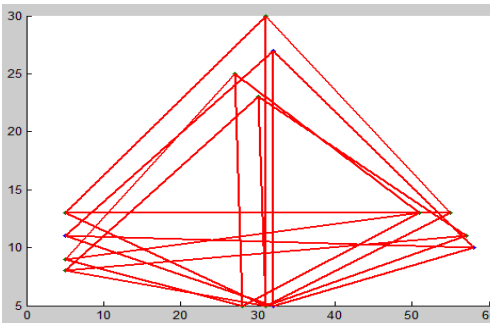

(d)

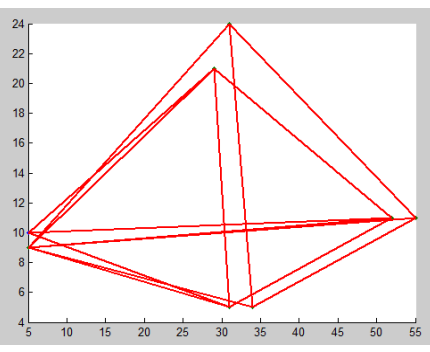

(b)

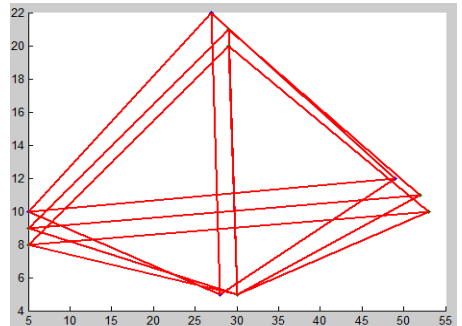

(e)

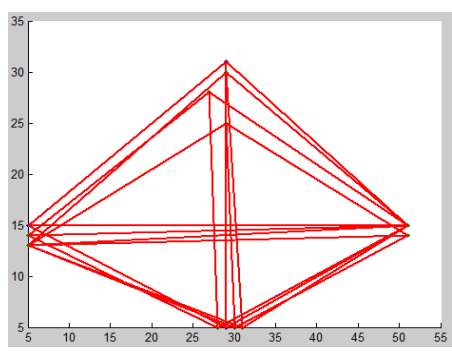

(c)

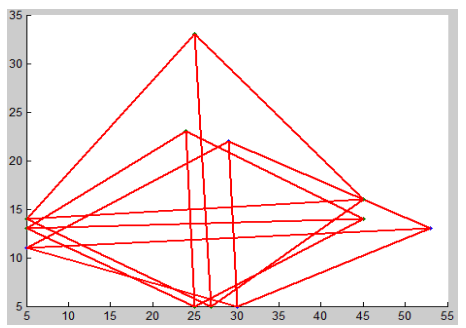

(f)

Figure 4. Defined quadrilateral shapes for different emotions from the JAFFE dataset: (a) Angry (KA.AN1 - KA.AN3), (b) Disgust (KA.DI1-KA.DI3), (c) Fear (KA.FE1KA.FE4), (d) Happy (KA.HA1-KA.HA4), (e) Sad (KA.SA1-KA.SA3), and (f) Surprise (KA.SU1-KASU3)

Furthermore, the quadrilateral shapes in geometry: square, rhombus, parallelogram, kite and isosceles trapezoid, are denoted as GeoQSs. Figure 4 shows that each $D Q S^{L}{ }_{e}$ in $G p D Q S^{L}{ }_{e}$ failed to match up with GeoQSs. However, the impreciseness of $D Q S^{L}{ }_{e}$ can be calculated using fuzzy membership functions. This can be done by calculating the contribution of the fuzzy functions of square, rhombus, parallelogram, kite and trapezoid in $D Q S^{L}{ }_{e}$. The contribution of $\mathrm{DQS}^{2}{ }_{2}$ in GeoQSs is shown in Figure 5, where the shapes in dotted lines represent the GeoQSs and the shapes drawn in continuous lines represent the defined shape. 
The proposed fuzzy membership functions for square, rhombus, parallelogram, kite and rectangle/isosceles trapezoid are presented in the following subsection, and the degrees of the functions are defined as:

$\mu_{c}(X)=\left\{\begin{array}{cl}\text { if } \mu_{c}(X)=0, & \text { Not a member of fuzzy set } \\ 0 \leq \mu_{c} \leq 1, & \text { Membe rship degree varies } \\ \mu_{c}(X)=1, & \text { Member of fuzzy set }\end{array}\right.$

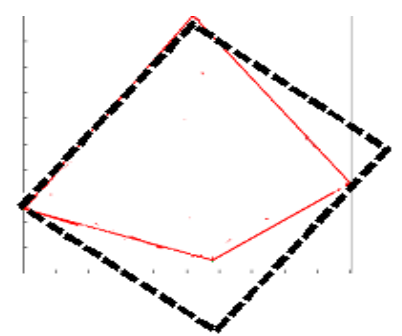

(a) Square vs defined shape

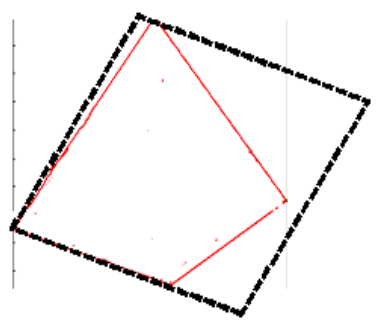

(b) Parallelogram vs defined shape

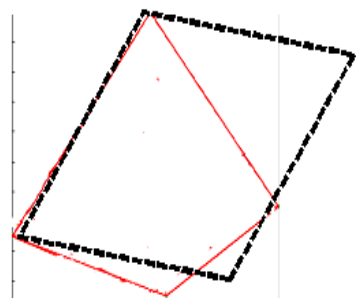

(c) Rhombus vs defined shape

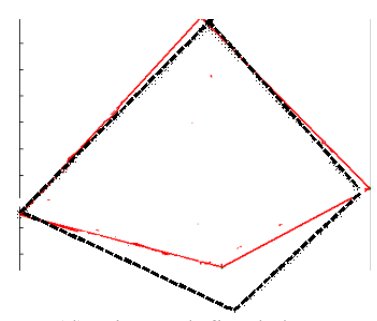

(d) Kite vs defined shape

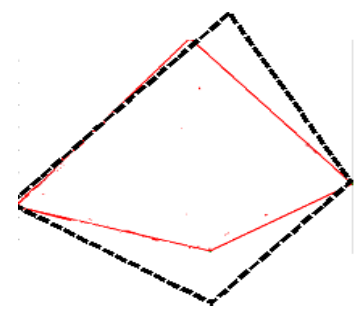

(e) Trapezoid vs defined shape

Figure 5. Geometrical shapes (dotted shapes) vs defined shape (continuous shapes)

Table 2. Six primitive shapes inferred from the MQS model

\begin{tabular}{|c|c|c|c|c|c|c|c|}
\hline Shape & Sides & Length1 & Width1 & Length2 & Width2 & Diagonal1 & Diagonal2 \\
\hline Square & $\mathrm{a}_{1} \mathrm{a}_{2} \mathrm{~b}_{1} \mathrm{~b}_{2}$ & $\mathrm{a}_{1} \mathrm{~b}_{1}$ & $\mathrm{a}_{1} \mathrm{a}_{2}$ & $\mathrm{a}_{2} \mathrm{~b}_{2}$ & $\mathrm{~b}_{1} \mathrm{~b}_{2}$ & $\mathrm{a}_{1} \mathrm{~b}_{2}$ & $\mathrm{a}_{2} \mathrm{~b}_{1}$ \\
\hline Rhombus & $\mathrm{a}_{1} \mathrm{a}_{3} \mathrm{~b}_{2} \mathrm{~b}_{4}$ & $\mathrm{a}_{1} \mathrm{~b}_{2}$ & $\mathrm{a}_{1} \mathrm{a}_{3}$ & $\mathrm{a}_{3} \mathrm{~b}_{4}$ & $\mathrm{~b}_{2} \mathrm{~b}_{4}$ & $\mathrm{a}_{1} \mathrm{~b}_{4}$ & $\mathrm{a}_{3} \mathrm{~b}_{2}$ \\
\hline Parallelogram & $\mathrm{a}_{1} \mathrm{a}_{4} \mathrm{~b}_{2} \mathrm{~b}_{5}$ & $\mathrm{a}_{4} \mathrm{~b}_{5}$ & $\mathrm{a}_{1} \mathrm{a}_{4}$ & $\mathrm{a}_{1} \mathrm{~b}_{2}$ & $\mathrm{~b}_{2} \mathrm{~b}_{5}$ & $\mathrm{a}_{1} \mathrm{~b}_{5}$ & $\mathrm{a}_{4} \mathrm{~b}_{2}$ \\
\hline Kite & $\mathrm{a}_{1} \mathrm{a}_{2} \mathrm{~b}_{1} \mathrm{c}_{1}$ & $\mathrm{a}_{2} \mathrm{c}_{1}(L)$ & $\mathrm{a}_{1} \mathrm{a}_{2}(\mathrm{~S})$ & $\mathrm{a}_{1} \mathrm{~b}_{1}(\mathrm{~S})$ & $\mathrm{b}_{1} \mathrm{c}_{1}(L)$ & $\mathrm{a}_{1} \mathrm{c}_{1}$ & $\mathrm{a}_{2} \mathrm{~b}_{1}$ \\
\hline Rectangle & $\mathrm{a}_{2} \mathrm{a}_{4} \mathrm{~b}_{3} \mathrm{~b}_{2}$ & $\mathrm{a}_{2} \mathrm{~b}_{2}$ & $\mathrm{a}_{2} \mathrm{a}_{4}$ & $\mathrm{a}_{4} \mathrm{~b}_{3}$ & $\mathrm{~b}_{2} \mathrm{~b}_{3}$ & $\mathrm{a}_{2} \mathrm{~b}_{3}$ & $\mathrm{a}_{4} \mathrm{~b}_{2}$ \\
\hline $\begin{array}{c}\text { Isosceles } \\
\text { trapezoid }\end{array}$ & $\mathrm{a}_{2} \mathrm{a}_{4} \mathrm{~b}_{5} \mathrm{~b}_{1}$ & $\mathrm{a}_{2} \mathrm{~b}_{1}$ & $\mathrm{a}_{2} \mathrm{a}_{4}$ & $\mathrm{a}_{4} \mathrm{~b}_{5}$ & $\mathrm{~b}_{1} \mathrm{~b}_{5}$ & $\mathrm{a}_{2} \mathrm{~b}_{5}$ & $\mathrm{a}_{4} \mathrm{~b}_{1}$ \\
& & & & & & & \\
\hline
\end{tabular}




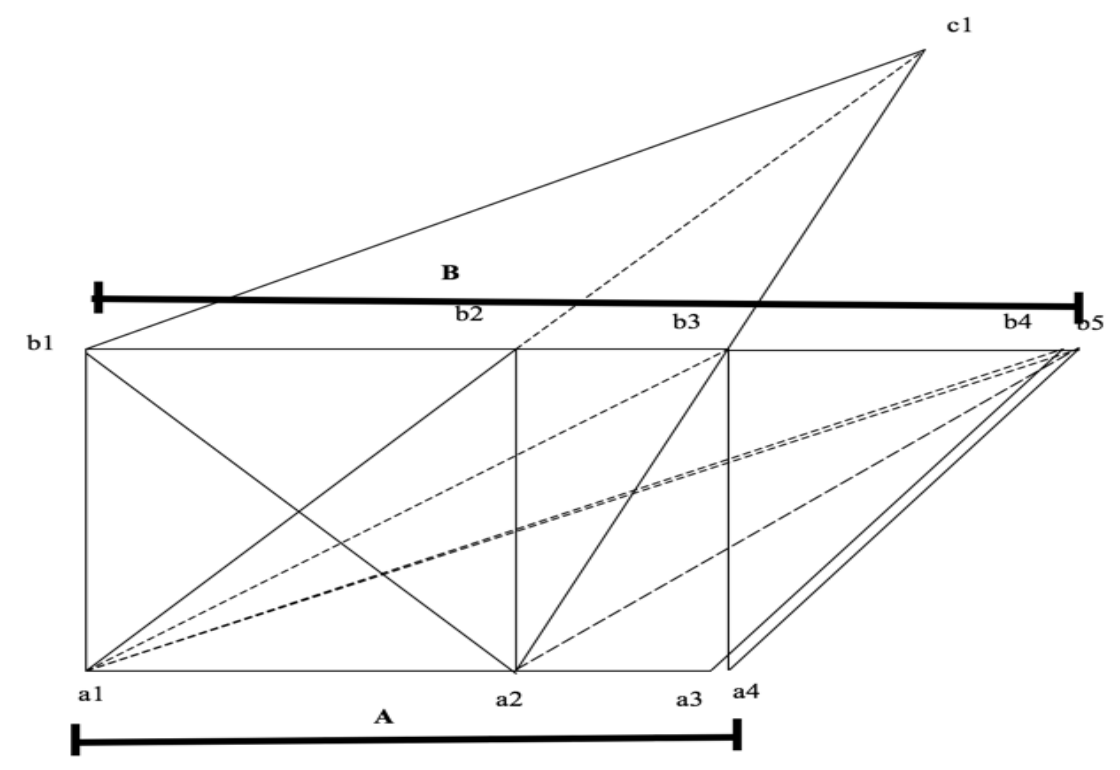

Figure 6. Variables used in the mixed quadratic shape model

\subsection{Fuzzy Membership Functions for the Defined Quadratic Shape}

As aforementioned, the proposed approach calculates the contribution of each GeoQSs in $D Q S^{L}$. The variables used in the proposed mixed quadratic shape model are shown in Figure 6 and in Table 2. In Figure 6, lines ' $A$ ' and ' $B$ ' indicate the widths of the MQSM and the vertical lines the lengths. Hence, $A$ as four points ' $a_{1}$ ', ' $a_{2}$ ',' $a_{3}$ ' and ' $a_{4}$ ', in the same way, $B$ as five points named as ' $b_{1}$ ', ' $b_{2},{ }^{\prime}, b_{3},{ }^{\prime}, b_{4}$ ' and ' $b_{5}$ '. The point ' $c_{1}$ ' is the starting point of the kite. The dotted lines between two endpoints are the diagonals of the respective shape. The proposed fuzzy membership functions are built as explained in the following subsections.

\subsubsection{Square}

In geometry, a four sided regular quadrilateral with all sides equal is called a square. A logical representation of a square is depicted in Figure 6 with $a_{1}, a_{2}, b_{2}$ and $b_{1}$. Based on the properties of this shape, the four equal lengths of the square are given by the distance between any two adjacent points, i.e. $a_{1} a_{2}=a_{2} b_{2}=b_{2} b_{1}=b_{1} a_{1}$. The length of the diagonals is the distance between opposite vertices: $a_{1} b_{2}=b_{1} a_{2}$. Only one of the diagonals is shown in Figure 6 to preserve the clarity of the diagram. It can be noted that the diagonal of any square is always greater than its side by $\sqrt{ } 2$ times; therefore, the fuzzy membership function for a perfect 
square is defined as:

$\left.\mu_{S q}(M Q S)=e^{\{-[M Q S} S q^{-1]^{2}}\right\}$

where $M Q S_{S q}=\left[\left(2 *\left(a_{1} b_{1}+a_{1} a_{2}\right)\right) /\left(a_{1} b_{2}+a_{2} b_{1}\right)\right]$, i.e. $M Q S_{S q}$ is defined as twice the ratio between the sum of two adjacent sides and the sum of the two diagonals. $M Q S_{s q}$ is assigned to each $C Q S$ of $G C Q S_{e}$ to check the perfectness of the square. Hence in Eq. (8) the values range from:

$\mu_{S q}(M Q S)= \begin{cases}1 \leq \mu \leq 1.4 & , \text { perfect square } \\ 0 \leq \mu<1 & , \forall \text { others }\end{cases}$

\subsubsection{Parallelogram}

The well-known property of a parallelogram (PP) is that both lengths and widths are different, as depicted in Figure 6 by $a_{1}, a_{4}, b_{2}$ and $b_{5}$. The lengths $b_{2} b_{5}$ and $a_{1} a_{4}$ are the upper and lower sides of parallelogram, and $a_{1} b_{2}$ and $a_{4} b_{5}$ are the widths. However, the opposite sides of the PP are equal, i.e. $b_{2} b_{5}=a_{1} a_{4}$ and $a_{1} b_{2}=a_{4} b_{5}$. The PP has a long diagonal and a short one: $a_{1} b_{5} \neq a_{4} b_{2}$. Using these properties, the fuzzy membership function for a perfect parallelogram is derived as:

$\left.\mu_{P l l}(M Q S)=e^{\{-[M Q S}{ }_{P l l}^{-1]^{2}}\right\}$

where $\operatorname{MQS}_{P l l}=\left[\left(\left(a_{4} b_{5}-a_{1} a_{4}\right) *\left(a_{1} b_{5}-a_{4} b_{2}\right)\right) /\left(\left(b_{2} b_{5}-a_{1} b_{2}\right) *\left(a_{4} b_{2}-a_{1} b_{5}\right)\right)\right]$, which is a ratio of two products where the difference of two adjacent sides and the difference of the two diagonals (with a change in sign) in both the numerator and the denominator and the values always range between $[0,1]$.

\subsubsection{Rhombus}

Rhombus is a special type of parallelogram with two diagonals: one long and one short. It differs from a parallelogram since all of its four sides have the same length. A logical 
representation of a rhombus with its diagonals is shown in Figure 6 by $a_{1}, a_{3}, b_{2}$ and $b_{4}$, with the lengths indicated by $a_{1} b_{2}=a_{1} a_{3}=a_{3} b_{4}=b_{2} b_{4}$ and the diagonals by $a_{1} b_{4} \neq a_{3} b_{2}$.

The membership value of a perfect rhombus is the ratio between two adjacent sides with the differences in the diagonals which is equals to 1 (one):

$$
\mu_{R h}(M Q S)=e^{\left\{-\left[M Q S_{R h^{-1}}\right]^{2}\right\}}
$$

where $\operatorname{MQS}_{R h}=\left[\left(a_{1} b_{2} *\left(a_{1} b_{4}-a_{3} b_{2}\right)\right) /\left(b_{2} b_{4} *\left(a_{1} b_{4}-a_{3} b_{2}\right)\right)\right]$.

\subsubsection{Kite}

A kite is a quadrilateral with four sides grouped into two sets of equal length sides which are adjacent to one another. Interestingly, a parallelogram also has two sets of equivalent length sides; however, they are opposite to one another. A pictorial representation of a kite is shown in Figure 6 by $a_{1}, a_{2}, b_{1}$ and $c_{1}$. The fuzzy membership function using the variables of a kite is given by Eq. 12, where $b_{1} c_{1}=a_{2} c_{1}$ and $b_{1} a_{1}=a_{1} a_{2}$ according to the property of the two segments joining opposite points of tangency of equal length; additionally, the diagonals connecting opposite ends have different lengths:

$\mu_{K t}=e^{\left\{\left[\frac{\left(b_{l} c_{1}-a_{1} a_{2}\right) *\left(a_{1} c_{1}-a_{2} b_{l}\right)}{\left(a_{1} b_{l}-a_{2} c_{l}\right) *\left(a_{2} b_{l}-a_{l} c_{l}\right)}-1\right]^{2}\right\}}$

\subsubsection{Rectangle and Isosceles trapezoid}

A rectangle is shown in Figure 6 by $a_{2}, b_{2}, b_{3}$ and $a_{4}$, where the diagonals are of equal length and the adjacent sides are not, i.e. $a_{2} b_{3}=b_{2} a_{4}$ and $a_{2} a_{4} \neq b_{2} b_{3}$. Figure 6 represents the rectangle pictorially that appears within the isosceles trapezoid. The isosceles trapezoid is typically considered a special type of rectangle as shown in Figure 6 by $a_{2}, b_{1}, b_{5}$ and $a_{4}$, where two opposite sides are parallel and the other two sides are of equal length, i.e. $b_{1} b_{5} / /{ }^{\mathrm{el}} a_{2} a_{4}$ and $a_{2} b_{1}=a_{4} b_{5}$, respectively, which means that adjacent sides do not have equal lengths. The diagonals split each other into similar regions with lengths that are pairwise equal. As 
pictured in Figure 6, the diagonals $b_{1} a_{4}=b_{5} a_{2}$ have the same length. Hence, the fuzzy membership function is derived for both rectangular and isosceles trapezoid as:

$$
\mu_{R / I t}=e^{\left\{\left[\frac{\left(a_{2} b_{3}\right) *\left(a_{2} b_{2}-a_{2} a_{4}\right)}{\left(a_{4} b_{2}\right) *\left(a_{4} b_{3}-b_{2} b_{3}\right)}-1\right]^{2}\right\}}
$$

or

$$
\mu_{R / I t}=e^{\left\{\left[\frac{\left(a_{2} b_{5}\right) *\left(a_{2} b_{1}-a_{2} a_{4}\right)}{\left(a_{4} b_{1}\right) *\left(a_{4} b_{5}-b_{1} b_{5}\right)}-1\right]^{2}\right\}}
$$

\subsection{Fuzzy Set}

A set $\left(\mu_{m}\right)$ of membership degrees obtained from Eqs. (8) - (13) for each $D Q S^{L}{ }_{e}$ of the different $e \in\{1,2, \ldots E\}$ generates the fuzzy set $E_{F S}$, where $m$ represents the total number of fuzzy membership functions. Each degree in $E_{F S}$ varies in the real unit interval of $[0,1]$, say $\mu_{m}: E_{F S} \rightarrow[0,1]$. Using the matrix of the fuzzy relation $E_{F R}: E_{F S} \rightarrow E_{S}$, which relates the membership degrees with its associated emotion, the classification decision can be achieved. $E_{F S}$ reveals that the minimum number of fuzzy values is used for predicting facial expression; hence, the required processing time, resources and storage are substantially reduced. Then, $E_{F R}$ is the input for a machine learning algorithm, which plays an important role to improve the classification accuracy. The proposed approach was tested using recent machine learning algorithms as described and discussed in the next section.

\section{Experimental results}

In this section, the recognition rates of the proposed approach are assessed and compared against other common approaches.

\subsection{Dataset}

The JAFFE and Cohn-Kanade Facial Expression $(\mathrm{CK}++)$ databases for facial 
expression analysis were used to assess the proposed and the other state-of-the-art approaches. JAFFE has 213 grayscale facial expression images (neutral - 30, angry - 30, disgust - 29, fear - 33, happy - 30, sad - 31, and surprise - 30) of ten subjects. This controlled database was taken under similar lighting conditions and without occlusion. All the images have a resolution of 256x256 pixels. As previously described, the three-quarters of the lower part of each input image were considered the ROI, from which the mouth region is extracted and used in the posterior processing steps. In addition, the $\mathrm{CK}++$ dataset available at $\mathrm{CMU}$, in Pittsburgh, USA (Kanade, Cohn, \& Tian 2000) was used, which consists of 593 image sequences from 123 subjects. The facial expressions in each grayscale sequence begin with a neutral face and increase to the height of the emotion given in the last frame. These peak expression frames from each sequence were used to validate the performance of the proposed approach. Experiments were carried out for the six emotions: anger, disgust, fear, happy, sad and surprise; however, the neutral images were discarded. Therefore, all images used in this study are public available and were acquired according to the Ethics Commissions of the related Institutions.

\subsection{Feature Extraction from Mouth}

The accuracy rate decreases substantially when the entire set of facial features is considered (Hernán F. García et al., 2016). Recently, researchers recognized emotions using important facial regions, particularly, the eyes and mouth (Ithaya Rani \& K. Muneeswaran et al., 2016). As discussed previously, the mouth provides more promising results than eyes, hence, the four vertices of the mouth in each input image of the JAFFE dataset were extracted, Figure 7.

Using the four vertices $A, B, C$ and $D$, the $D Q S^{L}{ }_{e}$ for all facial expression images were defined. Then, Eqs. (8) to (13) were applied on each $D Q S^{L}{ }_{e}$ to generate the $E_{F S}$. Each collection of membership degrees in $E_{F S}$ was mapped to the respective emotion to obtain the $E_{F R}$, and the recognition rate associated to each emotion was computed. 


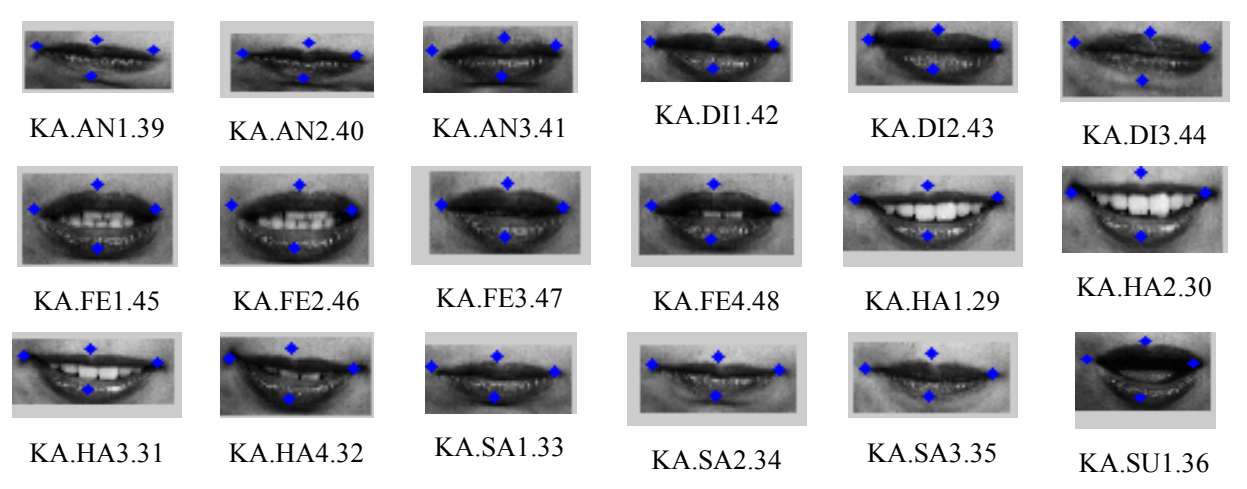

Figure 7. Cropped mouths from images indexed with "KA." in the JAFFE dataset with the four vertices highlighted

\subsection{Mouth against Eyes}

The highest recognition rates obtained by Gu et al., (2012) using the full faces on the JAFFE and $\mathrm{CK}++$ databases were $89.67 \%$ and $91.57 \%$, respectively. In this approach, the accuracy of the results was enhanced once again with the eyes/mouth occlusion. Three mask sizes, namely small, medium and large, were overlapped on the eyes and mouth and the results were evaluated. Based on the evaluation perform, it was concluded that the expressions with a masked mouth were more difficult to recognize than those with masked eyes. The results for the $\mathrm{CK}++$ database with large masks are given in Table 3, where there was a $12 \%$ improvement in the recognition rate for masked eyes.

Table 3. Results obtained by the method proposed by Gu et al. 2012 with mouth and eyes occluded with large masks

\begin{tabular}{llllllll}
\hline \hline Gu et al., 2012 & Happy & Sad & Surprise & Disgust & Angry & Scared & $\begin{array}{l}\text { Recognition } \\
\text { Rate }\end{array}$ \\
\hline Mouth Masked & 80.2 & 50.97 & 93.01 & 85.53 & 62.12 & 69.55 & $73.56 \%$ \\
Eyes Masked & $\mathbf{8 9 . 8}$ & $\mathbf{8 6 . 4 3}$ & $\mathbf{9 6 . 9 7}$ & $\mathbf{9 1 . 8 6}$ & $\mathbf{6 3 . 0 0}$ & $\mathbf{8 4 . 7 8}$ & $\mathbf{8 5 . 4 7 \%}$ \\
\hline \hline
\end{tabular}

Zhang Li et al. (2013) obtained a recognition rate of 78.6\% for lower AUs and of $71.3 \%$ for the upper ones. Moreover, in (Kotsia et al. 2009) the recognition accuracy obtained was 
$96.3 \%$ with eyes occlusion, and $93.7 \%$ with mouth occlusion. This concludes that the recognition accuracy for the mouth provides more promising outcomes than the eyes.

Table 4 presents the confusion matrices obtained by the proposed work and by the work presented in ( $\mathrm{Gu}$ et al. 2012) for the $\mathrm{CK}++$ dataset. The data in Table 4 shows that the recognition rate of the proposed approach was $96.5 \%$ and was $85.47 \%$ for the approach under comparison. This suggests that the proposed approach gives encouraging recognition rates compared to other similar works for the mouth, and that the mouth leads to more promising results than the eyes.

Table 4. Results with the $\mathrm{CK}++$ images by the proposed method and the one proposed by

Gu et al. 2012 only based on mouth features

\begin{tabular}{lccccccc}
\hline \hline Approach(mouth) & Angry & Disgust & Fear/Scared & Happy & Sad & Surprise & $\begin{array}{c}\text { Recognition } \\
\text { Rate }\end{array}$ \\
\hline Proposed work & $\mathbf{9 3 . 1}$ & $\mathbf{9 5 . 3}$ & $\mathbf{9 3 . 3 6}$ & $\mathbf{9 9 . 6 7}$ & $\mathbf{9 7 . 7}$ & $\mathbf{1 0 0}$ & $\mathbf{9 6 . 5 2 \%}$ \\
Gu et al. 2012 & 63.00 & 91.86 & 84.78 & 89.8 & 86.43 & 96.97 & $85.47 \%$ \\
\hline \hline
\end{tabular}

\subsection{Recognition Rate}

The local features extracted from the JAFFE images were analysed using the J48 decision tree to prune unwanted features from the dataset. Table 5 presents the confusion matrix obtained for the JAFFE dataset using Fuzzy Membership Functions (FMF) with the J48 decision tree algorithm. The Table includes the individual recognition percentage of each emotion along with the overall correct recognition percentage. The J48 decision tree algorithm is used to identify the essential features and reduces the outliers in each class. It greatly reduces both the input dimension and the required computational time. On using the J48 algorithm, the values of $\mu_{P l l}$ were found to be similar, which may not help in the discrimination of the emotions. Thus, the other four fuzzy features, namely $\mu_{S q}, \mu_{R h}, \mu_{K t}$ and $\mu_{R / I}$, were selected for further use in the machine learning algorithm to improve the accuracy rate. 
Table 5. Confusion matrix from the JAFFE dataset using the J48 algorithm

\begin{tabular}{llllllll}
\hline \hline & Angry & Disgust & Fear & Happy & Sad & Surprise & Percentage \\
\hline Angry & $\mathbf{2 6}$ & 1 & 1 & 0 & 2 & 0 & $\mathbf{8 6 . 6 6 \%}$ \\
Disgust & 2 & $\mathbf{2 0}$ & 2 & 1 & 4 & 0 & $\mathbf{6 8 . 9 9 \%}$ \\
Fear & 0 & 2 & $\mathbf{2 9}$ & 0 & 1 & 0 & $\mathbf{9 0 . 6 5 \%}$ \\
Happy & 0 & 0 & 0 & $\mathbf{3 1}$ & 0 & 0 & $\mathbf{1 0 0 \%}$ \\
Sad & 2 & 1 & 1 & 0 & $\mathbf{2 7}$ & 0 & $\mathbf{8 7 . 0 9 \%}$ \\
Surprise & 3 & 0 & 2 & 0 & 0 & $\mathbf{2 5}$ & $\mathbf{8 3 . 3 3 \%}$ \\
Average of Correctly Classified Instances: $86.12 \%$ & & \\
\hline \hline
\end{tabular}

The machine learning methods used in the facial emotion recognition were: SVM, MultiLayer Perceptron (MLP) and Ensemble learning, which have all been successfully used in other similar works. The parameters were selected separately for each classifier in order to find the best values that led to the highest classification. The LibSVM toolbox of the SVM was used with the Linear, Polynomial, Radial Basis Function (RBF) and Sigmoid kernels. Among these, the best result was obtained with the RBF kernel, and with the parameters $\mathrm{C}=$ 20 and gamma $=0.1$, see Table 6 . The MLP classifier presented good results with a topology of 4 neurons in the input layer, 20 neurons in the hidden layer and 7 in the output layer, and the learning rate $=0.3$, momentum $=0.2$ and training time $=500$. Finally, a recent machine learning technique, mainly ensemble learning, was used to assess the performance of the proposed approach. In the first model an Adaboost meta-algorithm with Random Forest was used, and in the second model, bagging with Hidden Markov Model was combined to build the model for prediction. In the random forest tree, resampling was applied as a preprocessing step, which is a supervised filter to produce a random subset of the input dataset. Each classifier was trained using 10-fold cross-validation. Using these configurations, the recognition accuracies of the JAFFE dataset for the different classifiers were computed and the confusion matrices were built, Tables 6-9. 
Table 6. Confusion matrix obtained with the JAFFE dataset using SVM with RBF

\begin{tabular}{ccccccccc}
\hline \hline & Angry & Disgust & Fear & Happy & Sad & Surprise & Percentage \\
\cline { 2 - 8 } & Angry & $\mathbf{3 0}$ & 0 & 0 & 0 & 0 & 0 & $\mathbf{1 0 0 \%}$ \\
Disgust & 1 & $\mathbf{2 8}$ & 0 & 0 & 0 & 0 & $\mathbf{9 6 . 6 \%}$ \\
Fear & 0 & 0 & $\mathbf{3 0}$ & 1 & 1 & 0 & $\mathbf{9 3 . 8 \%}$ \\
Happy & 0 & 0 & 0 & $\mathbf{3 0}$ & 1 & 0 & $\mathbf{9 6 . 8 \%}$ \\
Sad & 0 & 0 & 0 & 0 & $\mathbf{3 1}$ & 0 & $\mathbf{1 0 0 \%}$ \\
Surprise & 1 & 2 & 0 & 2 & 0 & $\mathbf{2 5}$ & $\mathbf{8 3 . 3 3 \%}$ \\
Average of Correctly Classified Instances: $\mathbf{9 5 . 0 8 \%}$ & & & \\
\hline \hline
\end{tabular}

Table 7. Confusion matrix obtained with the JAFFE dataset using MLP

\begin{tabular}{ccccccccc}
\hline \hline & Angry & Disgust & Fear & Happy & Sad & Surprise & Percentage \\
\cline { 2 - 7 } & Angry & $\mathbf{3 0}$ & 0 & 0 & 0 & 0 & 0 & $\mathbf{1 0 0 \%}$ \\
Disgust & 1 & $\mathbf{2 8}$ & 0 & 0 & 0 & 0 & $\mathbf{9 6 . 6 \%}$ \\
Fear & 0 & 0 & $\mathbf{3 1}$ & 0 & 1 & 0 & $\mathbf{9 6 . 9 \%}$ \\
Happy & 0 & 0 & 0 & $\mathbf{3 0}$ & 1 & 0 & $\mathbf{9 6 . 8 \%}$ \\
Sad & 0 & 0 & 0 & 0 & $\mathbf{3 1}$ & 0 & $\mathbf{1 0 0 \%}$ \\
Surprise & 1 & 2 & 0 & 2 & 0 & $\mathbf{2 5}$ & $\mathbf{8 3 . 3 \%}$ \\
Average of Correctly Classified Instances: $\mathbf{9 5 . 6 3 \%}$ & & \\
\hline \hline
\end{tabular}

Table 8. Confusion matrix obtained with the JAFFE dataset using the Bagging + HMM model

\begin{tabular}{ccccccccc}
\hline \hline & Angry & Disgust & Fear & Happy & Sad & Surprise & Percentage \\
\cline { 2 - 8 } & Angry & $\mathbf{3 0}$ & 0 & 0 & 0 & 0 & 0 & $\mathbf{1 0 0 \%}$ \\
Disgust & 1 & $\mathbf{2 7}$ & 0 & 0 & 1 & 0 & $\mathbf{9 3 . 1 \%}$ \\
Fear & 0 & 0 & $\mathbf{3 1}$ & 0 & 1 & 0 & $\mathbf{9 6 . 9 \%}$ \\
Happy & 0 & 0 & 0 & $\mathbf{3 0}$ & 1 & 0 & $\mathbf{9 6 . 8 \%}$ \\
Sad & 0 & 0 & 0 & 0 & $\mathbf{3 1}$ & 0 & $\mathbf{1 0 0 \%}$ \\
Surprise & 1 & 2 & 0 & 2 & 0 & $\mathbf{2 5}$ & $\mathbf{8 3 . 3 3 \%}$ \\
Average of Correctly Classified Instances: $\mathbf{9 5 . 0 8 \%}$ & & \\
\hline \hline
\end{tabular}


Table 9. Confusion matrix obtained with the JAFFE dataset using the Adaboost + Random Forest model

\begin{tabular}{ccccccccc}
\hline \hline & Angry & Disgust & Fear & Happy & Sad & Surprise & Percentage \\
\cline { 2 - 7 } & Angry & $\mathbf{3 0}$ & 0 & 0 & 0 & 0 & 0 & $\mathbf{1 0 0 \%}$ \\
Disgust & 1 & $\mathbf{2 7}$ & 0 & 0 & 1 & 0 & $\mathbf{9 3 . 1 \%}$ \\
Fear & 0 & 0 & $\mathbf{3 1}$ & 0 & 1 & 0 & $\mathbf{9 6 . 9 \%}$ \\
Happy & 0 & 0 & 0 & $\mathbf{3 0}$ & 1 & 0 & $\mathbf{9 6 . 8 \%}$ \\
Sad & 0 & 0 & 0 & 0 & $\mathbf{3 1}$ & 0 & $\mathbf{1 0 0 \%}$ \\
Surprise & 1 & 1 & 0 & 1 & 0 & $\mathbf{2 7}$ & $\mathbf{9 0 . 0} \%$ \\
Average Correctly Classified Instances: $\mathbf{9 6 . 1 7 \%}$ & & & \\
\hline \hline
\end{tabular}

Tables 6-9 show that all classifiers provided more or less the same outcome and efficiency: with an accuracy from 95.1 to $96.2 \%$. Beside the overall efficiency, the recognition rate of all classifiers was equal to $100 \%$ for the emotions angry and sad. The emotions that follow the highest recognition rates were happy and disgust. It seems that the recognition rates for the surprise class tend to be lower compared to the other emotions, as it was confused with the other expressions. The probable reason reported in (Zhang Li et al. 2013) for this finding is that the surprise images on the JAFFE database have a closed or only slightly open mouth. However, the proposed approach showed an interesting result for the surprise emotion using the random forest based ensemble learning classifier.

The consolidated results for the different classifiers in terms of average recognition rate using 10-fold cross validated and percentage split are shown in Table 10. 
Table 10. Average recognition rate of the proposed approach using different classifiers

\begin{tabular}{lll} 
Classifier & $\begin{array}{l}\text { Tenfold cross- } \\
\text { validated }\end{array}$ & $\begin{array}{l}\text { Training samples (66\%) } \\
\text { Testing samples (34\%) }\end{array}$ \\
\hline SVM & $95.08 \%$ & $96.8 \%$ \\
BAGGING + HMM & $95.08 \%$ & $97.1 \%$ \\
MLP & $95.63 \%$ & $\mathbf{9 9 . 8 \%}$ \\
ADABOOST + & $\mathbf{9 6 . 1 7 \%}$ & $98.4 \%$ \\
RANDOM FOREST & & \\
\hline
\end{tabular}

In terms of percentage split, the proposed approach was evaluated using the default values of the classifiers with $66 \%$ for training purposes and $34 \%$ for evaluation. Table 10 shows that the average accuracy rates obtained by the classifiers were always higher than $95 \%$ reaching a peak at $99.8 \%$. The proposed approach achieved the best accuracy rate $(99.8 \%)$ with percentage split, which is better than the ones obtained by the state-of-the-art approaches. However, the 10-fold cross-validator was the best estimator and the Adaboost+Random forest model obtained the best results among all the classifiers. Hence, the Adaboost + Random forest was chosen as the proposed model classifier.

Apart from the recognition rate, statistical tests are required to prove the performance of a new classification method. Thus, the statistical measures, namely, sensitivity (SEN), specificity (SPEC), positive predictive value (PPV), F-measures were calculated for our method applying all the classifiers used in this work, Table 11.

Table 11. Overall performance of the proposed approach using different classifiers

\begin{tabular}{lccccc}
\hline \hline CLASSIFIER & SEN & SPEC & PPV & F-MEASURE & TT \\
\hline \hline SVM & 0.951 & 0.99 & 0.954 & 0.95 & $\mathbf{0 . 1 8 s}$ \\
MLP & 0.956 & 0.991 & 0.958 & 0.956 & $0.64 \mathrm{~s}$ \\
BAGGING+ HMM & 0.951 & 0.99 & 0.953 & 0.950 & $0.33 \mathrm{~s}$ \\
ADABOOST + RANDOM & $\mathbf{0 . 9 6 2}$ & $\mathbf{0 . 9 9 2}$ & $\mathbf{0 . 9 6 4}$ & $\mathbf{0 . 9 6 2}$ & $0.35 \mathrm{~s}$ \\
FOREST & & & & & \\
\hline \hline
\end{tabular}


Sensitivity and specificity measure the percentage of positive and negative samples that are correctly recognized, respectively. PPV defines the proportion of positive outcomes in a statistical test. The data in Table 11 confirms that the overall averages were $95.5 \%$ for sensitivity, $99 \%$ for specificity, $95.5 \%$ for PPV and $95.5 \%$ for the F-Measure. These results show that the overall performance of the proposed approach was good. The, Random forest+Adaboost classifier achieved the best results followed by MLP, then the SVM classifier with the RBF kernel and the HMM based classifier which had similar performances. Furthermore, the proposed approach took only $0.18-0.64$ seconds (TT) to train the model; this high computational speed was due to fact that the model was trained using only four fuzzy features. Also, the proposed fuzzy membership functions used only the elementary arithmetical operations and operated over: the length, width and diagonals of the quadrilateral shapes, which takes a fixed computational time.

The Adaboost + Random forest model was found to outperform the other classifiers in both recognition rate and the statistical metrics. The performance of this classification model was assessed using the Receiving Operator Characteristics (ROC) curve, Figure 9. The X-axis of the ROC curve represents false positive (1-Specificity) and the Y-axis the true positive (Sensitivity). The best compromise is found when both sensitivity and specificity are highest at the same time. Further, the area under the ROC curve, called AUC, is 1 (one) for a perfect predictive power. Figure 8 shows that the best predictive power for all emotions was found using the Adaboost+Random forest classifier model. 


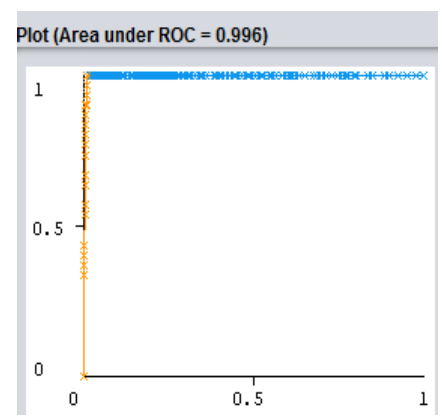

a. Angry

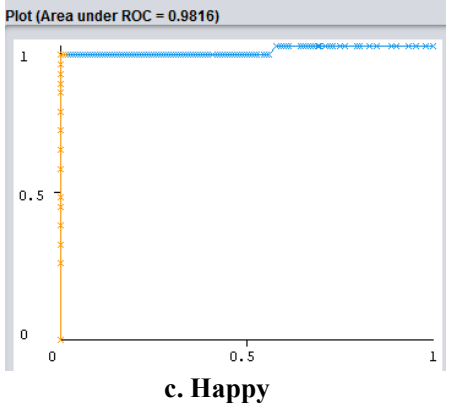

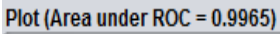

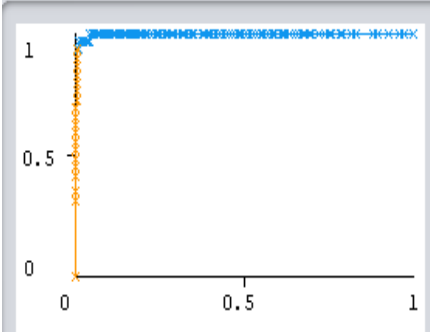

b. Disgust

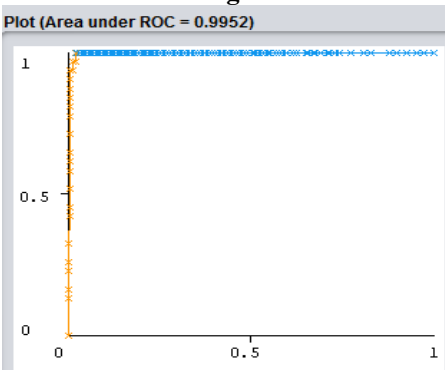

d. Sad

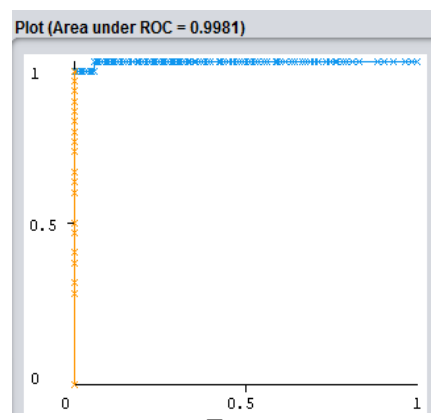

c. Fear

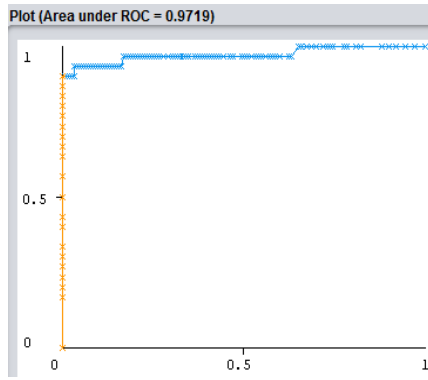

e. Surprise

Figure 8. ROC curves for all emotions using the Adaboost + Random forest classifier model

However, the ROC curves might be mislead when handling highly unbalanced datasets. Therefore, graphs were drawn for Precision versus Recall (PR) to interpret the performance of the proposed method in a more objective manner. Hence, the PR curves in Figure 9 indicate the number of true positives that are likely to be obtained in a competent predictive system.

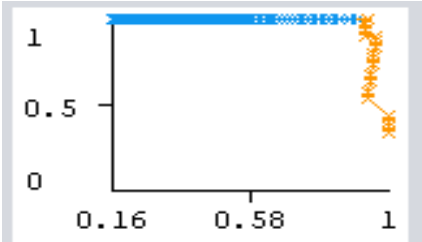

a. Angry

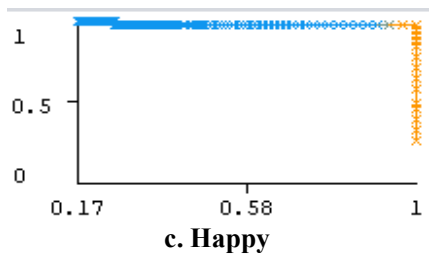

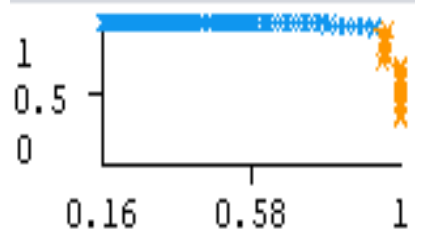

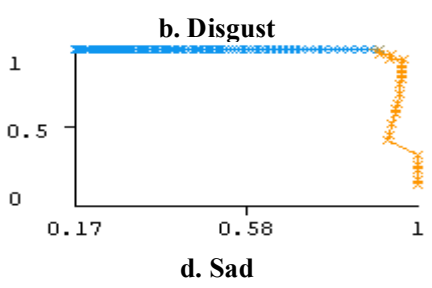

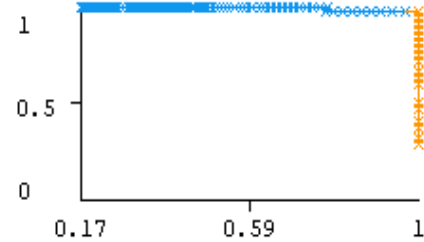

c. Fear

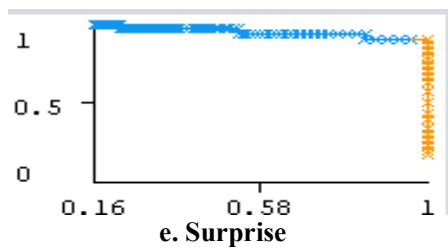

Figure 9. PR curves for all emotions when classified using the Adaboost + Random forest 
classifier model

Table 12. Results obtained by different state-of-the-art approaches when classifying the different emotions on the JAFFE database

\begin{tabular}{lccccccc}
\hline \hline \multicolumn{1}{c}{ Literature } & Angry & Disgust & Fear & Happy & Sad & Surprise & Average \\
\hline Proposed Work & $\mathbf{1 0 0}$ & $\mathbf{9 3 . 1}$ & $\mathbf{9 6 . 9}$ & $\mathbf{9 6 . 8}$ & $\mathbf{1 0 0}$ & $\mathbf{9 0 . 0}$ & $\mathbf{9 6 . 1 7 \%}$ \\
Vilas H Gaidhane et al., 2016 & 92.6 & 94.4 & 91.0 & 96.0 & 94.2 & 96.5 & $94.11 \%$ \\
Happy S L et al. 2015 & 100 & 86.2 & 93.8 & 96.8 & 77.4 & 96.7 & $91.81 \%$ \\
Hua Wang et al., 2014 & 84.2 & 87.2 & 78.1 & 96.3 & 92.4 & 96.1 & $89.0 \%$ \\
Rahulamathavan et al. 2013 & 96.7 & 93.1 & 93.8 & 93.5 & 90.3 & 93.3 & $93.45 \%$ \\
Zhang Shiqing et al. 2012 & 92.4 & 90.8 & 87.5 & 96.2 & 84.2 & 88.3 & $89.88 \%$ \\
Gu et al. 2012 & 93.3 & 86.2 & 75.0 & 100 & 93.3 & 96.7 & $90.75 \%$ \\
Ligang Zhang et al. 2011 & 96.7 & 90.0 & 93.8 & 93.6 & 93.6 & 90.0 & $92.92 \%$ \\
Wu. T. et al. 2010 & 83 & 68 & 67 & 88 & 78 & 88 & $78.66 \%$ \\
\hline \hline
\end{tabular}

Table 12 presents the confusion matrix for the methods under comparison, and the proposed approach outperformed all the other approaches, and without the need of a neutral image as reference. The recognition rate obtained by $\mathrm{Wu}$ T et al. (2010) was lower than $80 \%$ and poorer compared to all the other approaches. The performances obtained by $\mathrm{Li}$ Zhang et al. (2014) were between $80-81 \%$. The results obtained by Zhang Shiqing et al. (2012) and Gu et al. (2012) were greater than 90\%, and the performances as to happy emotion obtained by S L et al. 2015, Ligang Zhang et al., (2011) and Rahulamathavan et al. (2013) reached 90\%. Vilas H Gaidhane et al., (2016) more recently presented an approach that reached $94 \%$. In short, the proposed approach gave encouraging results compared to the other approaches. These encouraging results are because the impreciseness and vagueness in the shapes used to classify each emotion were built using fuzzy membership functions.

When the proposed method was evaluated with the $\mathrm{CK}++$ dataset, the default parameter values were used for the classifiers and the SVM with the RBF kernel classifier gave the best results. Table 13 gives the confusion matrices of the proposed method as well as the other 
methods under comparison for the $\mathrm{CK}++$ database. The recognition rates for the surprise and happy emotions obtained by the proposed method were higher than those of the other methods. Recently, Ghimire et al. (2017) and Gu et al. (2012) suggested that the results for anger, fear and sadness emotions in the $\mathrm{CK}++$ dataset were more similar than the ones obtained for happy and surprise emotions. The best results were obtained when they took two peak expression frames for anger, fear and sadness and one for each of the happy and surprise emotions. Finally, they achieved a $97.25 \%$ recognition rate. The results of our proposed approach were similar with a value of $98.32 \%$; however, we only used the last frame of each case in the input database. Zhang Li et al. (2013) reported that the surprise images in the $\mathrm{CK}++$ database have an exaggerated open mouth and are easily distinguished from the other emotions. Also, the results for the surprise and anger emotions contrast with those obtained with the JAFFE database. Table 13 shows that the average results obtained (98.32\%) are better than those obtained by the other methods. The highest accuracy obtained by the proposed approach was for the emotion of surprise and the lowest was for the emotion of anger.

Tables 14 and 15 indicate the performance obtained previously by similar works on facial emotion recognition using the JAFFE and $\mathrm{CK}++$ databases, respectively. In general, the literature considers that a performance comparison with other approaches may not be analysed directly because of differences in method, subjects, number of features, classifier, number of classes, number of images used as well as differences in partitioning the datasets. However, the results of each method can be analysed by taking the recognition result from their respective articles and tabulated as in Table 14. Among all the state-of-the-art methods, the proposed approach obtained very good recognition rates using the JAFFE database. Table 14 shows that the proposed approach reached a recognition rate of $96.17 \%$, which is significantly higher than the rates obtained by the other methods. The number of features used to recognize emotions in the literature varies from 11 to 185 . However, the proposed approach considers only four fuzzy features and, even so, attained a recognition accuracy of $96.17 \%$. 
Table 13. Results of various approaches in the classification of the different emotions from the $\mathrm{CK}++$ database

\begin{tabular}{lccccccc}
\hline \hline \multicolumn{1}{c}{ Approach } & Angry & Disgust & Fear & Happy & Sad & Surprise & Average \\
\hline Proposed Work & $\mathbf{9 5 . 3}$ & $\mathbf{1 0 0}$ & $\mathbf{9 5 . 8}$ & $\mathbf{1 0 0}$ & $\mathbf{9 8 . 8}$ & $\mathbf{1 0 0}$ & $\mathbf{9 8 . 3 2 \%}$ \\
Deepak Ghimire et al. 2017 & 97.5 & 96.7 & 96 & 100 & 96.7 & 100 & $97.81 \%$ \\
Swapna Agarwal et al., 2017 & 97.5 & 94.6 & 82.6 & 98.2 & 92.3 & 100 & $94.2 \%$ \\
Vilas H Gaidhane et al., 2017 & 94 & 98.7 & 93.1 & 99.6 & 94.5 & 99.7 & 96.47 \\
Hsieh et al., 2015 & 93.3 & 93.8 & 90.5 & 94.5 & - & 96.1 & 93.6 \\
Zhang Li et al. 2015 & 85 & 95 & 85 & 97 & 90 & 98 & $90.38 \%$ \\
Xiaorong Pu et al. 2015 & 75 & 94.6 & 68.6 & 97.7 & 88.9 & 92.5 & $89.37 \%$ \\
Happy S L. 2015 & 87.8 & 94.3 & 93.3 & 94.2 & 96.4 & 98.5 & $94.09 \%$ \\
Hua Wang et al., 2014 & 70.4 & 94.3 & 80 & 94.4 & 87 & 98 & $87.4 \%$ \\
Hsu et al., 2014 & 86.7 & 96.6 & 68.0 & 97.1 & 75.0 & 97.6 & $86.8 \%$ \\
Zhang L et al. 2013 & 90 & 83 & 65 & 80 & 60 & 77 & $75.83 \%$ \\
Gu et al. 2012 & 63 & 91.9 & 84.8 & 89.8 & 86.4 & 97 & $86.63 \%$ \\
Poursaberi et al. 2012 & 87.0 & 91.9 & 91 & 96.9 & 84.6 & 91.2 & 90.38 \\
Zhong L et al. 2012 & 71.4 & 95.3 & 81.1 & 95.4 & 88.0 & 98.3 & $88.26 \%$ \\
Zhang Ligang et al. 2011 & 87.1 & 90.2 & 92 & 98.1 & 91.5 & 100 & $93.14 \%$ \\
Zhao Xiaoming et al. 2011 & 97.6 & 94.2 & 99.6 & 95.5 & 89.8 & 97.2 & $95.66 \%$ \\
Jain et al, 2011 & 76.7 & 81.5 & 94.4 & 98.6 & 77.2 & 99.1 & $87.90 \%$ \\
Song et al, 2010 & 90.6 & 86.0 & 84.6 & 93.6 & 90.2 & 92.3 & $89.56 \%$ \\
Wu et al. 2010 & 82.9 & 67.7 & 66.7 & 87.7 & 78.4 & 87.9 & $78.55 \%$ \\
Shan et al. 2009 & 85.1 & 97.5 & 79.9 & 97.5 & 74.7 & 97.3 & $88.83 \%$ \\
Uddin M Z et al. 2009 & 82.5 & 97.5 & 95 & 100 & 92.5 & 92.5 & $93.33 \%$
\end{tabular}


Table 14. Recognition rates obtained by different approaches using the JAFFE database

\begin{tabular}{|c|c|c|c|c|}
\hline Approach & Method & $\begin{array}{l}\text { No. of } \\
\text { features }\end{array}$ & Images & Accuracy $(\%)$ \\
\hline Proposed Work & Fuzzy Geometry & 4 & $\begin{array}{c}184 \\
\text { (without } \\
\text { neutral) }\end{array}$ & $\begin{array}{l}96.17 \\
\text { (Cross-validation) }\end{array}$ \\
\hline $\begin{array}{l}\text { Hung-Hsu Tsai et al., } \\
2017\end{array}$ & HOG+U-LTP & N/A & & 95.71 \\
\hline Happy S L et al. 2015 & Appearance Feature & $\begin{array}{l}18 \text { facial } \\
\text { patches }\end{array}$ & 183 & 91.7 \\
\hline $\begin{array}{l}\text { Rahulamathavan et al. } \\
2013\end{array}$ & $\begin{array}{l}\text { LFDA (in the } \\
\text { encrypted domain) }\end{array}$ & 40 & 213 & $\begin{array}{l}94.37 \\
\text { (leave-one-out) }\end{array}$ \\
\hline Zhang S et al. 2012 & $\begin{array}{l}\text { Local Binary Pattern } \\
+ \text { LFDA }\end{array}$ & 11 & 213 & $\begin{array}{l}90.70 \\
\text { (leave-one-out) }\end{array}$ \\
\hline Gupta et al. 2011 & $\begin{array}{l}\text { Hybrid (discrete } \\
\text { cosine transform }+ \\
\text { Gabor filter }+ \\
\text { Wavelet transform }+ \\
\text { Gaussian distribution) }\end{array}$ & Unknown & 213 & $\begin{array}{l}93.40 \\
\text { (Conventional) }\end{array}$ \\
\hline Zhao and Zhang (2011) & $\begin{array}{l}\text { Local binary pattern } \\
\text { + KDIsomap }\end{array}$ & 20 & 213 & $\begin{array}{l}81.59 \\
\text { (Cross-validation) }\end{array}$ \\
\hline $\begin{array}{l}\text { Zhang and } \\
\text { Tjondronegoro (2011) }\end{array}$ & Patch-based Gabor & 185 & 203 & $\begin{array}{l}93.48 \\
\text { (leave-one-out) }\end{array}$ \\
\hline Gu et al. 2010 & $\begin{array}{l}\text { Radial encoded } \\
\text { Gabor jets }\end{array}$ & 49 & 213 & $\begin{array}{l}89.67 \\
\text { (Cross-validation) }\end{array}$ \\
\hline Kyperountas et al. 2010 & $\begin{array}{l}\text { Salient Feature } \\
\text { vectors }\end{array}$ & - & 213 & 85.92 \\
\hline
\end{tabular}

The proposed approach partitioned the used input database through tenfold cross-validation and, obtained a recognition accuracy of $96.17 \%$ using the JAFFE dataset. Hung-Hsu Tsai et al. (2017) achieved a recognition accuracy of $95.71 \%$, which is close to the one obtained by the proposed approach. Other approaches, like those proposed by Happy S L et al., (2015), Rahulamathavan et al. (2013), Zhang and Tjondronegoro (2011), Gupta et al. (2011) and 
Zhang et al. (2012), used leave-one-out and conventional approaches for the input dataset partition and achieved more than 90\% of recognition accuracy. Zhao and Zhang (2011) and $\mathrm{Gu}$ et al. (2010) suggested other approaches based on cross-validation and obtained performances below $90 \%$. Based on all these findings, the recognition rate of the proposed approach, which used minimal feature points and the best cross-validation estimator, is encouraging relative to the rates of the other current methods.

Table 15 compares the recognition rates obtained by the various approaches using the $\mathrm{CK}++$ database. The number of features used in the $\mathrm{CK}++$ database to recognize the expressions varies from 30 to 180 . Meanwhile, the four fuzzy features used in the proposed approach through partitioning the input dataset using cross-validation achieved the highest accuracy of 98.32\%. Deepak Ghimire et al. (2017a and 2017b) achieved an accuracy close to our result using 29 and 52 features, respectively. Zhao and Zhang (2011), Zhang and Tjondronegoro (2011) and Gu et al. (2010) used 30 to 180 features, obtaining 90-95\% of accuracy. Saeed et al. (2014) used a low number of facial key points, only 8, but they only reached an accuracy of $83 \%$. The other action units and appearance based methods proposed by Zhang L et al. 2015, Pu X et al. 2015 and Happy S L. 2015, achieved 89-94\% of accuracy. The recent classifiers used in (Mollahossein et al., 2016; Elaiwat et al., 2016; J. Li and E. Y. Lam et al., 2015; Siddiqi M H et al., 2015; Ghimire D et al., 2014; Liu M et al., 2014; Aifanti N et al., 2014) obtained accuracies of 93-97\%, and in (Bing-Fei Wu et al., 2017; D. M. Vo et al., 2016; Jung H et al., 2015; Cruz AC et al., 2014) of 70-89.9\%. 
Table 15. Results of recognition rates of the different approaches using the $\mathrm{CK}++$ database

\begin{tabular}{|c|c|c|c|}
\hline Approach & Method & No. of feature & Accuracy $(\%)$ \\
\hline Proposed Work & Fuzzy Geometry & 4 features & $\begin{array}{c}\text { 98.32\%(Cross- } \\
\text { validation })\end{array}$ \\
\hline $\begin{array}{l}\text { Deepak Ghimire et al., } \\
2017 \mathrm{a}\end{array}$ & LBP + NCM features & $\begin{array}{l}29 \text { local } \\
\text { features }\end{array}$ & $97.25 \%$ \\
\hline $\begin{array}{l}\text { Deepak Ghimire et al., } \\
2017 b\end{array}$ & Salient geometric features & $\begin{array}{l}52 \text { facial } \\
\text { patches }\end{array}$ & $98.30 \%$ \\
\hline Bing-Fei Wu et al., 2017 & GM + W-CR-AFM & - & $89.84 \%$ \\
\hline D. M. Vo et al., 2016 & AlexNet + SVM & - & $86.83 \%$ \\
\hline Mollahossein et al., 2016 & $\mathrm{CNN}$ & - & $93.2 \%$ \\
\hline Elaiwat et al., 2016 & Spatio temporal & - & $95.66 \%$ \\
\hline Jung H et al., 2015 & $\mathrm{CNN}$ & - & $80.6 \%$ \\
\hline $\begin{array}{l}\text { J. Li and E. Y. Lam et al., } \\
2015\end{array}$ & $\mathrm{CNN}$ & - & $96.8 \%$ \\
\hline Siddiqi M H et al., 2015 & $\begin{array}{c}\text { Stepwise linear discriminant } \\
\text { analysis }\end{array}$ & - & $96.83 \%$ \\
\hline Zhang L et al., 2015 & Action Unit & 56 features & $\begin{array}{c}90.38 \% \text { (Cross- } \\
\text { validation) }\end{array}$ \\
\hline Xiaorong Pu et al., 2015 & Active Appearance Model & - & $\begin{array}{c}89.37 \% \text { (Cross- } \\
\text { validation) }\end{array}$ \\
\hline Happy S L., 2015 & Appearance Feature & $\begin{array}{l}18 \text { facial } \\
\text { patches }\end{array}$ & $94.1 \%$ \\
\hline Ghimire D et al., 2014 & HOG feature, ELM Ensemble & - & $97.30 \%$ \\
\hline Liu M et al., 2014 & spatio-temporal & - & $94.19 \%$ \\
\hline Saeed A et al., 2014 & $\begin{array}{c}\text { Geometric features, SVM } \\
\text { classifier }\end{array}$ & $\begin{array}{c}8 \text { facial key } \\
\text { points }\end{array}$ & $83.01 \%$ \\
\hline Cruz AC et al., 2014 & Temporal features & - & $71.83 \%$ \\
\hline Aifanti N et al., 2014 & Facial key point tracking & - & $94.31 \%$ \\
\hline Zhao and Zhang (2011) & LBP + KDIsomap & 30 features & $\begin{array}{l}94.88 \text { (Cross- } \\
\text { validation) }\end{array}$ \\
\hline Zhang and Tjondronegoro & Patch-based Gabor & 180 features & 94.48 (Leave-one-out) \\
\hline
\end{tabular}


(2011)

Gu et al. (2010)

\section{Conclusion}

In this article, a new approach based on a minimum number of features extracted from the mouth region and without using a reference face was proposed to recognize human emotions. The extracted features were used to draw a quadrilateral for the face under analysis and the associated degree of impreciseness was addressed using the proposed mixed quadratic shape model through fuzzy membership functions. The proposed fuzzy membership functions were square, rhombus, kite and isosceles trapezoid. To validate the proposed approach, common learning methods were used to classify the human emotions and their recognition rates compared. The best recognition rates of the proposed approach were $96.17 \%$ and $98.32 \%$ for the JAFFE and CK++ datasets, respectively, and which were comparatively higher than the ones obtained by other recently proposed approaches.

The major advantages of the proposed approach are: only four facial features, fuzzy membership functions and fuzzy features are used to accurately identify the human emotions under evaluation here. The development of the proposed model based only on four fuzzy features reduced the computation time and space. The detection of the emotions without the need of a reference face brings this computational approach closer to the human system of perception. Finally, the evaluation with a competent cross validator and with statistical tests confirmed the efficiency of our approach.

A future work will continue the proposed method but will also consider other factors like, age and gender, which also play vital roles in emotion recognition.

\section{Acknowledgments}

João Manuel R.S. Tavares gratefully acknowledges the funding of Project NORTE-010145-FEDER-000022 - SciTech - Science and Technology for Competitive and Sustainable 
Industries, co-financed by "Programa Operacional Regional do Norte" (NORTE2020), through "Fundo Europeu de Desenvolvimento Regional” (FEDER).

\section{Conflict of interest statement}

The authors report no conflict of interest.

\section{References}

1. Anderson K, McOwan P. W (2004) Robust real-time face tracker for use in cluttered environments. Computer Vision and Image Understanding, 95(2), pp. 184-200.

2. Anima Majumder, Laxmidhar Behera, Venkatesh K. Subramanian (2014) Emotion recognition from geometric facial features using self-organizing map. Pattern Recognition, 47, pp. 1282-1293.

3. Aifanti N, Delopoulos A (2014) Linear subspace for facial expression recognition. Signal Process Image Commun, 29, pp. 177-18

4. Asthana A, Saragih J, Wagner M, Goecke R (2009) Evaluating AAM fitting methods for facial expression recognition. In Proceeding of the international conference on affective computing and intelligent interaction, pp. 1-8

5. Bartlett M, Littlewort G, Frank M, Lainscsek C, Fasel I, Movellan J (2005) Recognizing facial expression :machine learning and application to spontaneous behavior. In: IEEE Computer Society Conference on Computer Vision and Pattern Recognition, vol. 2, pp. 568573.

6. Bing-Fei Wu, and Chun-Hsien Lin (2017) Adaptive Feature Mapping for Customizing Deep Learning Based Facial Expression Recognition Model. IEEE access 2017.

7. Barthomeuf L, Droit-Volet S, Rousset S (2012) How emotions expressed by adults' faces affect the desire to eat liked and disliked foods in children compared to adults. British Journal of Developmental Psychology, Vol. 30, pp. 253-266.

8. Belhumeur P, Hespanha J, Kriegman D (1997) Eigenfaces vs. fisher face: recognition using class specific linear projection. IEEE Transactions on Pattern Analysis and Machine Intelligence, 19(7), pp. 711-720. 
9. Cruz AC, Bhanu B, Thakoor NS (2014) Vision and attention theory based sampling for continuous facial motion recognition. IEEE Trans Affect Comput, 5, pp. 418-431

10. Deng H. B, Jin L. W, Zhen L. X, Huang J. C (2005) A new facial expression recognition method based on local Gabor filter bank and PCA plus LDA. International Journal of Information Technology, 11(11), pp. 86-96.

11. Deepak Ghimire, Joonwhoan Lee, Ze-Nian Li and Sunghwan Jeong (2017a) Recognition of facial expressions based on salient geometric features and support vector machines. Int. J. of Multimed Tools Appl, 76, pp. 7921-7946.

12. Deepak Ghimire, Sunghwan Jeong, Joonwhoan Lee and San Hyun Park (2017b) Facial expression recognition based on local region specific features and support vector machines. Multimed Tools Appl, 76, pp. 7803-7821.

13. Donato G, Bartlett M. S, Hager J C, P. Ekman, T. J. Sejnowski, Classifying facial actions, IEEE Transactions on Pattern Analysis and Machine Intelligence, 1999, 21(10), pp. 974-989.

14. Ekman P, Friesen W (1978) Action Coding System: A Technique for the Measurement of Facial Movement. Palo Alto: Consulting Psychologists Press.

15. Elaiwat S, Bennamoun M, and Boussaid F (2016) A spatio-temporal RBM-based model for facial expression recognition. Int. J of Pattern Recognition, vol. 49, pp. 152-161.

16. Feng X.Y, Hadid A, Pietikainen M (2005) Facial expression recognition with local binary patterns and linear programming. Pattern Recognition and Image Analysis, 15(2), pp. $546-548$.

17. Feng X.Y., Hadid A, Pietikainen M (2004) A coarse-to-fine classification scheme for facial expression recognition. In: The First International Conference on Image Analysis and Recognition, pp. 668-675.

18. Ghimire D, Lee J (2013) Geometric feature-based facial expression recognition in image sequences using multi-class AdaBoost and support vector machines. Sensors 13(77), pp. 14 7734 
19. Ghimire D, Lee J (2014) Extreme learning machine ensemble using bagging for facial expression recognition. J Inf Process Syst 10(3), pp. 443-458

20. Gu W, Venkatesh Y, Xiang C (2010) A novel application of self-organizing network for facial expression recognition from radial encoded contours. SoftComput. Fusion Found. Methodol. Appl., 14(2), pp. 113-122.

21. Gupta S.K, Agrwal S, Meena Y. K, Nain N (2011) A hybrid method of feature extraction for facial expression recognition. In: 7th international conference on signal image technology \& internet-based systems, pp. 422-425.

22. Happy S L and Aurobinda Routray (2015) Automatic Facial Expression Recognition Using Features of Salient Facial Patches. IEEE Transactions on Affective Computing, Vol 6(1), pp. 1-12.

23. He L.H, Zou C.R, Zhao L, Hu D (2005) An enhanced LBP feature based on facial expression recognition. In: IEEE Engineering in Medicine and Biology 27th Annual Conference, pp. 3300-3303.

24. Heisele B, Ho P, Wu J, Poggio T (2003) Face recognition: component-based versus global approaches. Computer Vision and Image Understanding, 91, pp. 6-21.

25. Hernán F. García, Mauricio A. Álvarez, Álvaro A. Orozco (2017) Dynamic facial landmarking selection for emotion recognition using Gaussian processes, In: Journal on Multimodal User Interfaces, 11, pp. 327-340.

26. Hsieh CC, Hsih MH, Jiang MK, Cheng YM, Liang EH (2015) Effective semantic features for facial expressions recognition using svm. Int $\mathrm{J}$ of Multimedia Tools and Applications, pp. 1-20.

27. Hsu FS, Lin WY, Tsai TW (2014) Facial expression recognition using bag of distances. Int $\mathrm{J}$ of Multimedia Tools and Applications, 73(1), pp. 309-326.

28. Hua Wang, Heng Huang, Fillia Makedon (2014) Emotion Detection via Discriminant Laplacian Embedding, Univ Access Inf Soc, 13, pp. 23-31. 
29. Hung-Hsu Tsai, Yi-Cheng Chang (2017) Facial expression recognition using a combination of multiple facial features and support vector machine. Int. J of Soft Computing, DOI 10.1007/s00500-017-2634-3.

30. Ilbeygi M, Hosseini H. S (2012) A novel fuzzy facial expression recognition system based on facial feature extraction from color face images, Engineering Applications of Artificial Intelligence. 25, pp. 130-146.

31. Irene Kotsia, Ioannis Pitas (2007) Facial Expression Recognition in Image Sequences Using Geometric Deformation Features and Support Vector Machines. IEEE Transactions on Image Processing, 16(1), pp. 172-187.

32. Irene Kotsia, Ioan Buciu, Ioannis Pitas (2008) An analysis of facial expression recognition under partial facial image occlusion. Image and Vision Computing, 26, pp. 10521067.

33. Ithaya Rani1 P and Muneeswaran K (2017) Recognize the facial emotion in video sequences using eye and mouth temporal Gabor features. In: J of Multimed Tools Appl, 76, pp. 10017-10040.

34. Jung H, Lee S, Park S, Kim B, Kim J, Lee I and Ahn C (2015) Development of deep learning-based facial expression recognition system. 21st Korea-Japan Joint Workshop on Frontiers of Comput. Vision (FCV), pp. 1-4, 2015.

35. Jain S, Hu C, Aggarwal C K (2011) Facial expression recognition with temporal modeling of shapes. In: IEEE International Conference on Computer Vision Workshops (ICCVWorkshops), Barcelona, pp. 1642-1649.

36. Juan M. Mayor Torres and Evgeny A. Stepanov (2017) Enhanced face/audio emotion recognition: video and instance level classification using ConvNets and restricted Boltzmann Machines. In: Proceedings of the International Conference on Web Intelligence, ACM.

37. Kazmi S. B, Qurat-ul-Ain, Jaffar M. A (2012) Wavelet-based facial expression recognition using a bank of support vector machines. Soft Computing, 16(3), pp. 369-379. 38. Kharat G. U, Dudul S. V (2009) Emotion recognition from facial expression using neural networks. In: Human-computer systems interaction, AISC 60, pp. 207-219. 
39. Keith Anderson, Peter W McOwan (2006) A Real-Time Automated System for the Recognition of Human Facial Expressions. IEEE Transactions on Systems, Man, and Cybernetics-Part B: Cybernetics, 36(1), pp. 96-105.

40. Kotisa I, Pitas I (2007) Facial expression recognition in image sequence using geometric deformation features and support vector machines. IEEE Trans Image Process 16:172-187.

41. Kobayashi H, Hara F (1997) Facial interaction between animated 3dface robot and human beings. In: IEEE International Conference on Systems, Man, and Cybernetics. Computational Cybernetics and Simulation, IEEE, vol. 4, pp. 3732-3737.

42. Lanitis A, Taylor C, Cootes T (1997) Automatic interpretation and coding of face images using flexible models. IEEE Trans. Pattern Anal. Mach. Intell., 19, pp. 743-756.

43. Liu W. F, Yi S. J, Wang Y. J (2009) Automatic facial expression recognition based on local binary patterns of local areas. In: WASE international conference on information engineering, pp. 197-200.

44. Liu C, Wechsler H (2003) Independent component analysis of Gabor features for face recognition. IEEE Transactions on Neural Network, 14(4), pp. 919-928.

45. Li J and Lam E. Y (2015) Facial expression recognition using deep neural networks. In Proc. IEEE Int. Conf. Imaging Syst. Techn. (IST), pp. 1-6.

46. Liu M, Shan S, Wang R, and Chen X (2014) Learning expression lets on spatio-temporal manifold for dynamic facial expression recognition. In Proc. IEEE Conf. Comput. Vis. Pattern Recognit. (CVPR), Columbus, OH, USA, 2014, pp. 1749-1756.

47. Luo R, Huang C, Lin P (2011) Alignment and tracking of facial features with component-based active appearance models and optical flow. In: International Conference on Advanced Intelligent Mechatronics(AIM), IEEE, pp. 1058 -1063.

48. Luo Y, Wu C-M, Zhang Y (2013) Facial expression recognition based on fusion feature of PCA and LBP with SVM, International Journal for Light and Electron Optics, 2013, 124(17), pp. 2767-2770.

49. A. Mehrabian (1968) Communication without words. Psychology Today, 2(4), pp. $53-$ 56. 
50. Mollahosseini A, Chan D and Mahoor M. H. (2016) Going deeper in facial expression recognition using deep neural networks. In Proc. IEEE Winter Conf. Appl. Comput. Vis. (WACV), Lake Placid, NY, USA, 2016, pp. 1-10.

51. Moore S, Bowden R (2011) Local binary patterns for multi-view facial expression recognition. Comput VisImage Underst 115:541-558

52. Lyons M, Budynek J, Akamatsu S (1999) Automatic classification of single facial images. IEEE Transactions on Pattern Analysis and Machine Intelligence, 21, pp. 13571362 .

53. Michel F. Valstar, Maja Pantic (2012) Fully Automatic Recognition of the Temporal Phases of Facial Actions. IEEE Transactions on Systems, Man, and Cybernetics-PART B: Cybernetics, 42(1), pp. 28-43.

54. Mingli Song, Dacheng Tao, Zicheng Liu, Xuelong Li, Mengchu Zhou (2010) Image Ratio Features for Facial Expression Recognition Application. IEEE Transactions on Systems, Man, And Cybernetics-Part B: Cybernetics, 40(3), pp. 779-788.

55. Moore S, Bowden R (2009) The effects of pose on facial expression recognition. In A. Cavallaro, S. Prince, D. Alexander (Eds.), In: Proceedings of the British machine conference, BMVA Press, pp. 79.1-79.11.

56. Nielsen J. A, Zielinski B. A, Ferguson M. A, Lainhart J. E, Anderson J. S. (2013) An evaluation of the left-brain vs. right brain hypothesis with resting state functional connectivity magnetic resonance imaging. PLOS One. Cognitive NeuroscienceConnectomics. DOI: 10.1371/journal.pone.0071275 Featured in PLOS Collections.

57. Ahmad Poursaberi et al., (2012) Gauss-Laguerre wavelet textural feature fusion with geometrical information for facial expression identification. EURASIP Journal on Image and Video Processing, https://doi.org/10.1186/1687-5281-2012-17.

58. Pablo Barros et al. (2017) Emotion-modulated attention improves expression recognition: A deep learning model. Neurocomputing, 253, pp. 104-114.

59. Padgett C, Cottrell G (1996) Representing face image for emotion classification. Advances in Neural Information Processing Systems, vol. 9, pp. 894-900. 
60. Paweł Tarnowski et al. (2017) Emotion recognition using facial expressions. In: International Conference on Computational Science - ICCS 2017, 12-14 June 2017, Zurich, Switzerland.

61. Qirong Mao et al. (2017) Learning emotion-discriminative and domain-invariant features for domain adaptation in speech emotion recognition. Speech Communication 93, pp. 1-10.

62. Rahulamathavan Y, Phan R. C-W, Chambers J. A, Parish D. J (2013) Facial expression recognition in the encrypted domain based on local fisher discriminant analysis. IEEE Transactions on Affective Computing, 4(1), pp. 83-92.

63. Rizwan Ahmed Khana, Alexandre Meyer, Hubert Konik, Saïda Bouaka (2013) Framework for reliable, real time facial expression recognition for low resolution images. Pattern Recognition Letters, 34, pp. 1159-1168.

64. Rudovic O, Pantic M, Patras I (2012) Coupled Gaussian processes for pose-invariant facial expression recognition. IEEE Trans Pattern Anal Mach Intell 25:1357-1369

65. Rosenblum M, Yacoob Y, Davis L (1996) Human expression recognition from motion using a radial basis function network architecture. IEEE Trans. Neural Netw., 7(5), pp. 11211138 .

66. Sarah Adel Bargal, Emad Barsoum, Cristian Canton Ferrer, Cha Zhang (2016) Emotion recognition in the wild from videos using images. In: ICM: Proceedings of the 18th ACM International Conference on Multimodal Interaction, ACM.

67. Shan C, Gong S, McOwan P.W (2005) Robust facial expression recognition using local binary patterns. In: IEEE International Conference on Image Processing, pp. 370-373.

68. Shan C, Gong S, McOwan P (2009) Facial expression recognition based on local binary patterns: a comprehensive study. Image Vis. Comput., 27(6), pp. 803-816.

69. F.Y. Shih, C. F. Chuang, P. S. P. Wang, Performance comparisons of facial expression recognition in JAFFE database. International Journal of Pattern Recognition, 2008, 22(3), pp. $445-459$. 
70. Saeed A, Al-Hamadi A, Niese R, Elzobi M (2014) Frame-based facial expression recognition using geometric features. Adv Hum Comput Interact, pp. 1-13

71. Siddiqi M. H, Ali R, Khan A. M, Park Y.-T, and Lee S (2015) Human facial expression recognition using stepwise linear discriminant analysis and hidden conditional random fields. IEEE Trans. Image Process., vol. 24, no. 4, pp. 1386-1398.

72. Swapna Agarwal, Dipti Prasad Mukherjee (2017) Facial expression recognition through adaptive learning of local motion descriptor. Int. J. of Multimed Tools Appl, 76, pp. 1073 1099

73. Sohail A.S.M, Bhattacharya P (2011) Classifying facial expressions using level set method based lip contour detection and multi-class support vector machines. Int. J. Pattern Recognit. Artif. Intell., 25(06), pp. 835-862.

74. Song M, Tao D, Liu Z, Li X and Zhou M(2010) Image ratio features for facial expression recognition application. IEEE Transactions on Systems, Man, and Cybernetics, Part B: Cybernetics, vol. 40, no. 3, pp. 779-788.

75. Sophie Jarlier, Didier Grandjean, Sylvain Delplanque, Karim N'Diaye, Isabelle Cayeux, Maria Inés Velazco, David Sander, Patrik Vuilleumier, and Klaus R. Scherer (2011) Thermal Analysis of Facial Muscles Contractions. IEEE Transactions on Affective Computing, 2(1), pp. 2-9.

76. Stefanos Zafeiriou and Ioannis Pita (2008) Discriminant Graph Structures for Facial Expression Recognition. IEEE Transactions on Multimedia, 10(8), pp. 1528-1540.

77. Thiago H.H. Zavaschi, Alceu S. Britto Jr., Luiz E.S. Oliveira, Alessandro L. Koerich (2013) Fusion of feature sets and classifiers for facial expression recognition. Expert Systems with Applications, 40, pp. 646-655.

78. Turk M, Pentland A (1991) Eigenfaces for recognition. Journal of Cognitive Neuroscience, 3(1), pp. 71-86.

79. Uddin M, Lee J, Kim T (2009) An enhanced independent component-based human facial expression recognition from video. IEEE Trans Consum Electron 55:2216-2224. 
80. Valstar M, Patras I, Pantic M (2005) Facial action unit detection using probabilistic actively learned support vector machines on tracked facial point data. In: IEEE Computer Society Conference on Computer Vision and Pattern Recognition, CVPR Workshops, pp. 76. 81. Vadivel A, Shanthi P, and Shaila S.G (2015) Estimating Emotions Using Geometric Features from Facial Expressions. Encyclopedia of Information Science and Technology, Third Edition, pp. 8.

82. Vilas H Gaidhane1, Yogesh V and Vijander Singh (2016) Emotion recognition using eigenvalues and Levenberg-Marquardt algorithm-based classifier. Indian Academy of Sciences, Vol. 41, No. 4, pp. 415-423.

83. Vo D. M and Le T. H. (2016) Deep generic features and SVM for facial expression recognition. 3rd National Foundation for Science and Technology Development Conf. Inf. and Comput. Sci. (NICS), pp. 80-84.

84. Wang Z, Ruan Q (2010) Facial expression based orthogonal local fisher discriminant analysis. In: Proc. ICSP 2010, pp. 1358-1361.

85. Wan Ding, Mingyu $\mathrm{Xu}$, Dongyan Huang, Weisi Lin, Minghui Dong, Xinguo $\mathrm{Yu}$, Haizhou Li (2016) Audio and face video emotion recognition in the wild using deep neural networks and small datasets. In: ICMI 2016: Proceedings of the 18th ACM International Conference on Multimodal Interaction, ACM.

86. Whitehill J, Bartlett M, Movellan J (2008) Automatic facial expression recognition for intelligent tutoring systems. In: Proc of Computer Vision and Pattern Recognition Workshops, Anchorage, AK, USA.

87. Wu T, Bartlett M, Movellan J.R (2010) Facial expression recognition using Gabor motion energy filters. In: Proceedings of IEEE Computer Society Conference on Computer Vision and Pattern Recognition Workshops (CVPRW'10), pp. 42-47.

88. Xiang C, Fan X. A, Lee T. H (2006) Face recognition using recursive fisher linear discriminant. IEEE Transactions on Image Processing, 15(8), pp. 2097-2105. 
89. Xiaorong Pu, Ke Fan, Xiong Chen, Luping Ji, Zhihu Zhou (2015) Facial expression recognition from image sequences using twofold random forest classifier. Neurocomputing 168, pp. 1173-1180

90. Xudong Xie, Kin-Man Lam (2009) Facial expression recognition based on shape and texture. Pattern Recognition, 42, pp. 1003-1011.

91. Yeasin M, Bullot B, Sharma R (2006) Recognition of facial expressions and measurements of levels of interest from video. IEEE Trans Multimed 8:500-508

92. Yuchi Huang and Hanqing Lu (2016) Deep learning driven hypergraph representation for image-based emotion recognition. In: Proceedings of the 18th ACM International Conference on Multimodal Interaction, ACM.

93. Zhang L, Tjondronegoro D (2011) Facial expression recognition using facial movement features. IEEE Transactions of Affective Computing, 2(4), pp. 219-229.

94. Zhang S, Zhao X, Lei B (2012) Robust facial expression recognition via compressive sensing. Sensors 12 , pp. 3747-3761

95. Zhang, X. Zhao, B. Lei (2012) Facial expression based on local binary patterns and local fisher discriminant analysis. WSEAS Transactions on Signal processing, 8(1), pp. 21-31.

96. Zhang Z, Lyons M, Schuster M, Akamatsu S (1998) Comparison between geometrybased and Gabor-wavelets - based facial expression recognition using multi-layer perceptron. In: Proceedings of 3rd International Conference on Automatic Face and Gesture Recognition, pp. 454-459.

97. Zhang Z (1999) Feature-based facial expression recognition: sensitivity analysis and experiments with a multi layer perceptron. Int. J. Pattern Recognit. Artif. Intell., 13, pp. 893911.

98. Zhao X, Zhang S (2011) Facial expression recognition based on local binary patterns and kernel discriminant isomap. Sensors, pp. 9573-9588.

99. Zhao G.Y, Pietikainen M (2007) Dynamic texture recognition using local binary patterns with an application to facial expressions. IEEE Transactions on Pattern Analysis and Machine Intelligence, 29(6), pp. 915-928. 
100. Zou J, Ji Q, Nagy G (2007) A comparative study of local matching approach for face recognition. IEEE Transactions on Image Processing, 16(10), pp. 2617-2628.

101.Zhang L, Jiang M, Farid D, Hossain A.M (2013) Intelligent facial emotion recognition and semantic- based topic detection for a humanoid robot. Expert Syst. Appl., 40(13), pp. $5160-5168$.

102. Zhang L and Tjondronegoro D (2011) Facial expression recognition using facial movement features. IEEE Transactions on Affective Computing, vol. 2, no. 4, pp. 219-229. 103.Li Zhang, Ming Jiang, Dewan Farid and M.A. Hossain (2013) Intelligent facial emotion recognition and semantic-based topic detection for a humanoid robot. Expert Systems with Applications, 40, pp. 5160-5168.

104. Li Zhang, Kamlesh Mistry, Ming Jiang, Siew Chin Neoh, Mohammed Alamgir Hossain (2015) Adaptive facial point detection and emotion recognition for a humanoid robot. Computer Vision and Image Understanding, 140, pp. 93-114.

105. Zhong L, Liu Q, Yang P, Liu B, Huang J and Metaxas D. N (2012) Learning active facial patches for expression analysis. In IEEE Conference on Computer Vision and Pattern Recognition (CVPR), Providence, RI, USA. 